\title{
Electrical control over the Fe(II) spin crossover in a single molecule: Theory and experiment
}

\author{
V. Meded, A. Bagrets, K. Fink, and R. Chandrasekar \\ Institute of Nanotechnology, Karlsruhe Institute of Technology, D-76021 Karlsruhe, Germany
}

\author{
M. Ruben* \\ Institute of Nanotechnology, Karlsruhe Institute of Technology, D-76021 Karlsruhe, Germany and \\ Institute de Physique et Chimie de Materiaux de Strasbourg (IPCMS), UMR 7504 CNRS-Université de Strasbourg, \\ F-67034 Strasbourg, France \\ F. Evers ${ }^{\dagger}$ \\ Institute of Nanotechnology, Karlsruhe Institute of Technology, D-76021 Karlsruhe, Germany and \\ Institut für Theorie der Kondensierten Materie, Karlsruhe Institute of Technology, D-76128 Karlsruhe, Germany
}

\author{
A. Bernand-Mantel, J. S. Seldenthuis, A. Beukman, and H. S. J. van der Zant ${ }^{\ddagger}$ \\ Kavli Institute of Nanoscience, Delft University of Technology, P.O. Box 5046, NL-2600 GA, The Netherlands \\ (Received 6 December 2010; revised manuscript received 8 March 2011; published 22 June 2011)
}

\begin{abstract}
We report on theoretical and experimental work involving a particular molecular switch, an $\left[\mathrm{Fe}^{\mathrm{II}}(\mathrm{L})_{2}\right]^{2+}$ complex, that utilizes a spin transition ("crossover"). The hallmark of this transition is a change of the spin of the metal ion, $S_{\mathrm{Fe}}=0$ to $S_{\mathrm{Fe}}=2$, at fixed oxidation state of the Fe ion. Combining density functional theory and first principles calculations, we demonstrate that within a single molecule this transition can be triggered by charging the ligands. In this process the total spin of the molecule, combining metal ion and ligands, crosses over from $S=0$ to $S=1$. Three-terminal transport through a single molecule shows indications of this transition induced by electric gating. Such an electric field control of the spin transition allows for a local, fast, and direct manipulation of molecular spins, an important prerequisite for molecular spintronics.
\end{abstract}

DOI: 10.1103/PhysRevB.83.245415

\section{INTRODUCTION}

In molecular electronics the challenge is condensing the functionality of single molecules into electronic devices, thus exploiting the versatility offered by chemical diversity for technology. Different from silicon-based devices, molecules can be synthesized with built-in functionality such as switching and rectifying behavior or magnetism. ${ }^{1-15}$ However, how to address this functionality electrically remains open. For ensembles of molecules in solution, electrochemistry is used to gain electrical access to redox states by applying an electrode potential to the buffer solution. Access of redox states of an individual molecule can be gained in planar three-terminal devices, which are fabricated on top of silicon substrates., ${ }^{2,5}$ Interestingly, at low temperatures these molecular junctions often exhibit Coulomb-blockade physics, which allows for a detailed analysis of spectroscopic features. For example, vibrational modes of the molecule ${ }^{5,16}$ or magnetic excitations ${ }^{17-20}$ have been probed for different charge states.

In addition to these generic features, molecules tend to have symmetries that are usually accompanied with spectral degeneracies which in turn can give rise to certain instabilities. A particularly intriguing magnetomechanical instability is the "spin transition" or "spin crossover" (ST). ${ }^{21}$ It has been studied in bulk molecular crystals for a long time; ${ }^{22}$ it is also an important topic in material science where it has been observed, for example, in oxide-material $\mathrm{LaCoO}_{3} .{ }^{23}$

The ST is related to the splitting $\Delta_{\text {oct }}$ of the $d$ multiplet of a magnetic ion inside a crystal field with octahedral symmetry (see Fig. 1). The $\Delta_{\text {oct }}$ splitting competes with the spin pairing energy of the $d$ electrons $U$, and it is known that it can be modified, for example, by light, pressure, or temperature. ${ }^{24-28}$
PACS number(s): 85.65.+h, 85.75.-d, 75.80.+q, 75.75.-c

At large octahedral splitting the metal ion finds itself in a low spin state (LS), for example, $S_{\mathrm{Fe}}=0$, while at smaller splitting a high spin state (HS), for example, $S_{\mathrm{Fe}}=2$, prevails.

With an eye on transport experiments, we have investigated the possibility of also triggering the ST by charging the molecule. Indeed, we predict that a specific model system $\left[\mathrm{Fe}^{\mathrm{II}}(\mathrm{bpp})_{2}\right]^{2+}$ [bpp: 2, 6-bis(pyrazol-1-yl) pyridine, see Fig. 2] undergoes a ST when two electrons are added. We argue that an experimental fingerprint to accompany this transition could be a split zero bias (Kondo) resonance in the current voltage characteristics $(I V)$. The split-Kondo peak indicates a zero field splitting of the triplet ground state with a magnitude roughly comparable to the Kondo scale $T_{\mathrm{K}}$. Indeed, a splitting of the Kondo resonance is observed in our experimental test which could therefore constitute a first report on the ST observed at a single molecule level. We further note that recently an impressive demonstration of mechanical control over spin-spin interactions has been demonstrated by Parks et al. ${ }^{29}$ who were able to tune the spin-orbit coupling by application of strain. Also in that transport experiment, a split zero-bias resistance ("Kondo") peak served as the hallmark for experimental verification.

We emphasize that the ST described here differs in an important way from phenomena observed in earlier experiments on quantum dots ${ }^{30}$ metal clusters, and molecules. ${ }^{31}$ In these cases magnetism is typically related to the exchange interaction between the two valence electrons of the quantum dot. In the case of the spin crossover compounds discussed in this paper the valence electrons are located on the ligands. Our calculations show that these two valence electrons only interact weakly with each other if the Fe core is in the low spin state. 


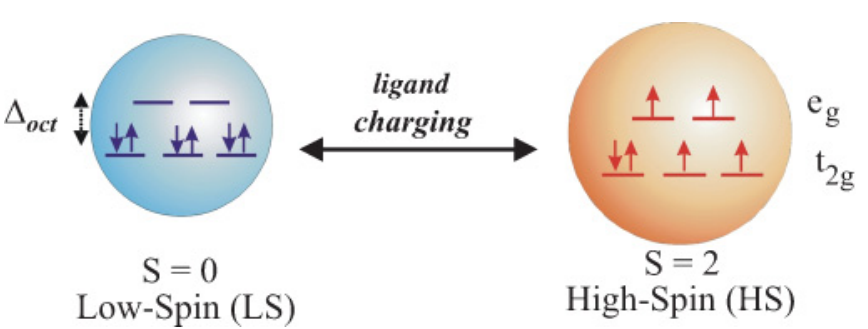

FIG. 1. (Color online) Schematic representation of the spin transition of octahedrally coordinated $\mathrm{M}^{\mathrm{II}}$ metal ions: two different $d^{6}$ electronic configurations with closed (low spin, LS) and open shell (high spin, HS) are accessible. The orbital population depends on the octahedral energy splitting $\Delta_{\text {oct }}$, which is due to the electronic Coulomb repulsion and controlled by the ligand system.

The observed large Kondo splitting, however, indicates that the charging of the two ligands triggers the ST transition. Thus, the ST results from an interaction-induced interplay between the valence electrons on the ligands and the $d$ electrons in the lower energy shells located near the $\mathrm{Fe}^{\mathrm{II}}$ core. Altogether eight electrons and seven orbitals are involved.

\section{THEORY: ANIONS IN THE GAS PHASE}

Our theoretical analysis begins with formulating the computational model that we employ for calculating the groundstate energy (DFT level) of the molecule $\mathrm{Fe}^{\mathrm{II}}(\mathrm{bpp})_{2}^{2+}$.

\section{A. Model formulation and computational method}

A typical microscopic realization underlying transport experiments in molecular electronics is depicted in Fig. 2. Since the metal ion is charged, countercharges will be located in the vicinity of the molecular core; their detailed arrangement depends on the gating method. In Fig. 2 we indicated a cylindrical screening geometry, which is mimicking an electrochemical environment. ${ }^{32-34}$ In the investigations presented in this section countercharges have been ignored. We study their quantitative effect on magnetic excitation spectra in Appendix D.

Quite generally, ignoring countercharges is justified when focusing on situations where the size of the screening cloud well exceeds the extension of the ligand system. Under such conditions, the cloud's screening potential is almost constant inside the molecular complex so that its main consequence is shifting the orbital energy spectrum against the vacuum level. Such potentials do not give rise to forces and therefore their impact on structural properties of the ground state should be negligible.

\section{B. Ground states from density functional theory}

The calculations presented in this section are based on the density functional theory (DFT) using a real-space based package TURBOMOLE. ${ }^{35}$ The generalized gradient approximation (GGA) functional BP86, ${ }^{36}$ the meta-GGA based hybrid functional $\mathrm{TPSSH}^{37}$ and the hybrid functional B3LYP ${ }^{38}$ were used in unrestricted open shell calculations with a def2-TZVP basis set. ${ }^{39,40}$ Since magnetomechanical effects are essential, a careful geometry optimization is mandatory. The key parameter is the (averaged) metal-nitrogen bond length

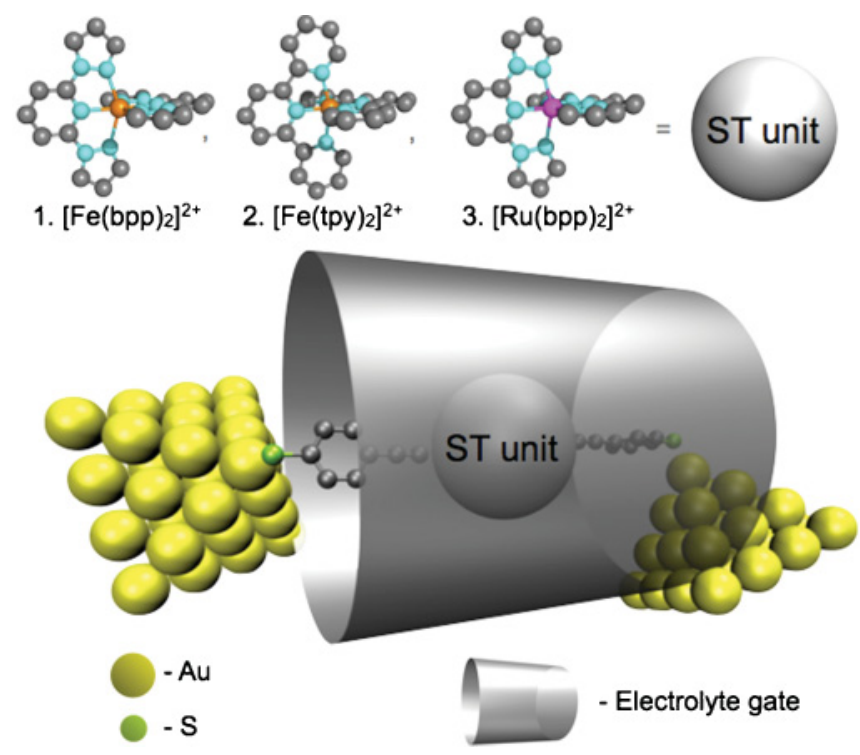

FIG. 2. (Color online) Top: Atomic structure of the complexes $\mathbf{1}\left(\left[\mathrm{Fe}(\mathrm{bpp})_{2}\right]^{2+}\right)$, bpp-bispyrazolyl pyridine $\mathbf{2}\left(\left[\mathrm{Fe}(\mathrm{tpy})_{2}\right]^{2+}\right)$, tpyterpyridine and $\mathbf{3}\left(\left[\mathrm{Ru}(\mathrm{bpp})_{2}\right]^{2+}\right)$. (The molecular complex used in the experiment has in addition to $\mathbf{1}$ pyridine anchor groups.) Colors: dark gray: carbon; cyan (light gray): nitrogen; red (gray): iron; magenta (gray): ruthenium; hydrogen atoms are not displayed. Bottom: model geometry of a single molecule transport measurement. ${ }^{8,13,32-34}$ The surrounding cylinder models an electrolyte gate.

$d_{\mathrm{av}}=\left(d_{\mathrm{on}}+2 d_{\text {off }}\right) / 3$ (see Fig. 3 for definitions), which is significantly shorter at the optimized structure of the LS state compared to the HS state (see Fig. 4). Which one of the two has the lower energy depends on control parameters, most notably the gate potential.

\section{Molecule with charge $Q=+2$}

Employing density functional calculations we have determined the total energy in each of the two local minima and thus have identified the true ground state with respect to the charge state of the molecule. As may be inferred from Table I, we have found that the $\mathrm{Fe}^{\mathrm{II}}$ complex without excess charge $(Q=+2)$ has lowest energy in its closed shell, LS, configuration with the HS state being $\Delta E \approx 0.5-1 \mathrm{eV}$ above. Our results for the excitation energy into the HS
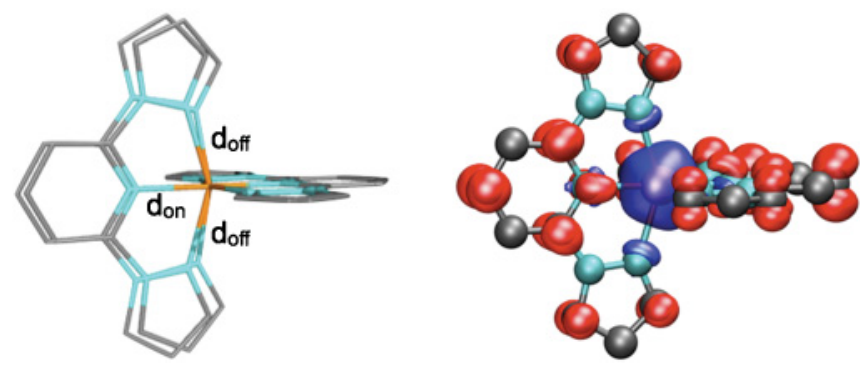

FIG. 3. (Color online) Metal-organic complex $\mathrm{Fe}^{\mathrm{II}}(\mathrm{bpp})_{2}^{2+}$ : Left: low spin (narrow) and high spin (wide) geometries ( $\mathrm{Fe}^{\mathrm{II}}$ : red (dark gray), C: gray, N: turquoise(light gray)). Right: Spin density of the molecule with two additional electrons. The plot highlights the antiferromagnetic couplings between the excess electrons (located on the ligands, red) and the high spin core (blue). 


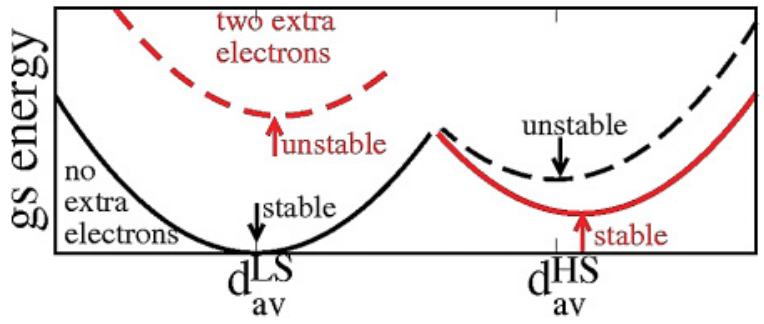

FIG. 4. (Color online) Schematic representation illustrating the dependency of the energy of the $\mathrm{Fe}^{\mathrm{II}}(\mathrm{bpp})_{2}^{2+}$ complex on the geometry, here represented by the (averaged) distance between the $\mathrm{Fe}^{\mathrm{II}}$ ion and the ligand based $\mathrm{N}$ atoms. Black lines: the complex at $Q=+2$, no excess charge. Red lines: same complex with two extra electrons, $Q=0$.

state, $\Delta E=E(\mathrm{HS})-E(\mathrm{LS})$, have an uncertainty due to the approximations in the exchange-correlation (XC) functionals (BP86, TPSSH, and B3LYP) underlying the DFT calculations. A rough upper bound for the error thus induced may be found by comparing $\Delta E$ obtained for the GGA (Table I, upper two data rows), with the result obtained with hybrid functionals, TPSSH and B3LYP (Table I, lower four data rows). Indeed, with B3LYP a HS state appears to be more favorable, indicated by a weakly negative $\Delta E$, but only slightly so. We take this as a confirmation of the general trend. ${ }^{41}$

\section{Molecule with charge $Q=0$}

By contrast, when we consider additional electrons on the molecule, calculations indicate that the situation is reverse: for the case of two extra electrons $Q=0$, LS becomes unstable against a HS ground state with $S=2$. Again, referring to Table I, for the GGA and TPSSH we witness a change in the sign of $\Delta E$ indicating a (first order) transition from the LS to the HS ground state. The critical charge might be close to $Q_{\text {crit }}=+1$. The B3LYP functional fully confirms the trend toward a stabilization of the magnetic ground state with $Q$ going from +2 to 0 . Conversely, we conclude that the critical charge, where the ST occurs for B3LYP should be near $Q_{\text {crit }}=+3$. The situation is summarized in Fig. 4. The change in bond length associated with the metal ion expansion is typically $10 \%$; the value compares well with experimental measurements for the bulk material (see Appendix C, Table VI). To illustrate this effect, Fig. 3 (left) displays the overlay of the two molecular conformations in the LS and HS state.

Importantly, the expansion of the core region is of a magnetomechanical origin and is not related to a charge injection into the $\mathrm{Fe}$ core. This conclusion is drawn from detailed analysis of the computed charge density profile near the metal ion: the net charge of the $\mathrm{Fe}^{\mathrm{II}}$ is $Q \approx+2$, that is, not changed under the transition. Alternatively, one can also analyze the spin density, which we display in Fig. 3 (right). The localized character of the up-spin configuration with integrated spin density $S_{\mathrm{Fe}} \approx 1.75$ near the core region clearly shows the HS state of $\mathrm{Fe}^{\mathrm{II}}$. As may also be inferred from Table I, the Mulliken spin population is $S \approx 1.8-1.9$ in the HS state nearly independent of the charge $Q$.

The residual coupling between the core and the excess electrons is antiferromagnetic, so that the spin density distributed over the ligands is down-spin [see Fig. 3 (right)] adding up to a total spin of the molecule $S=1$. The ligands thus represent two very weakly coupled reduced ligands with spin $1 / 2$ in the presence (HS) or absence (LS) of the Fe $\mathrm{Fe}^{\mathrm{II}}$-based spin $S_{\mathrm{Fe}}=2$. (We provide further arguments supporting these findings in Appendix A.)

\section{Ground state and excitations from wave function based methods (CASSCF and MCCEPA)}

In this section we investigate more deeply into the spin structure of the high spin ground states. We will see that the triplet ground state of HS is separated by a large excitation gap $(87 \mathrm{meV}$ ) from a higher lying quintet state. Due to spinorbit interaction the triplet ground state exhibits a zero field splitting. The corresponding excitation energy $\delta E$ will be in the milli-electron-volt range ${ }^{42}$ and depends strongly on the detailed microscopic structure of the molecular junction.

\section{Ground state}

We investigated the energy difference between the high spin and the low spin state of the $Q=+2$ system by CASSCF (complete active space self consistent field) ${ }^{43,44}$ and MCCEPA (multiconfiguration coupled electron-pair approximation) ${ }^{45}$ calculations. For the $Q=+2$ system the basis set dependence of the energy difference of the high spin and the low spin state of complex 1 was determined in the individual geometries obtained in the DFT calculations with the TPSSH functional.

TABLE I. Data for complex 1 [Fe(bpp) $]^{2+}$ (Fig. 3) showing the evolution of molecular properties with increasing molecular charge $Q=+2,0$ : total energy difference $\Delta E=E(\mathrm{HS})-E(\mathrm{LS})$ between HS and LS conformations ( $\Delta E>0$ means LS is the ground state), average Fe-N bond length $d_{\mathrm{av}}^{\mathrm{HS}, \mathrm{LS}}$, local spin moment $S$ on Fe (defined through the half of the difference between spin-up and spin-down electron populations on metal core after the standard Mulliken analysis), and values for the spin gap $\Delta_{\text {oct }}$. Data are presented for the three XC functionals used in DFT calculations: BP86, ${ }^{36}$ TPSSH, ${ }^{37}$ and B3LYP. ${ }^{38}$

\begin{tabular}{|c|c|c|c|c|c|c|c|}
\hline \multirow[b]{2}{*}{$Q$} & \multirow[b]{2}{*}{$\mathrm{XC}$} & \multirow[b]{2}{*}{$\Delta E(\mathrm{eV})$} & \multicolumn{2}{|c|}{$\left[\mathrm{Fe}(\mathrm{bpp})_{2}\right]^{2+} \mathrm{HS}$} & \multicolumn{3}{|c|}{$\left[\mathrm{Fe}(\mathrm{bpp})_{2}\right]^{2+} \mathrm{LS}$} \\
\hline & & & $d_{\mathrm{av}}^{\mathrm{HS}}(\AA)$ & $S(\mathrm{Fe})$ & $d_{\mathrm{av}}^{\mathrm{LS}}(\AA)$ & $S(\mathrm{Fe})$ & $\overline{\Delta_{\text {oct }}(\mathrm{eV})}$ \\
\hline+2 & BP86 & 1.065 & 2.19 & 1.89 & 1.95 & 0.0 & 2.75 \\
\hline 0 & BP86 & -0.242 & 2.14 & 1.67 & 1.94 & 0.21 & 2.81 \\
\hline+2 & TPSSH & 0.490 & 2.19 & 1.92 & 1.96 & 0.0 & 4.54 \\
\hline 0 & TPSSH & -1.084 & 2.16 & 1.765 & 1.96 & 0.0 & 4.38 \\
\hline+2 & B3LYP & -0.134 & 2.22 & 1.91 & 2.0 & 0.0 & 5.74 \\
\hline 0 & B3LYP & -1.606 & 2.19 & 1.79 & 1.99 & 0.0 & 5.77 \\
\hline
\end{tabular}


TABLE II. $\Delta E=E(\mathrm{HS})-E(\mathrm{LS})$ in electron volts for different sets of polarization functions $\left(\left[\mathrm{Fe}(\mathrm{bpp})_{2}\right]^{2+}\right.$ complex, $\left.Q=+2\right)$. Exponents of the polarization function were taken from the TURBOMOLE catalog. Fe was equipped by a Wachters basis set, ${ }^{74}$ the Fe coordinating $\mathrm{N}$ atoms by a Huzinaga $11 s 7 p$ basis set, ${ }^{75}$ and the polarization functions given above, all other atoms by a Huzinaga DZ basis set.

\begin{tabular}{lcccc}
\hline \hline Basis & CASSCF & MCCEPA & Fe & $\mathrm{N}$ \\
\hline Basis 1 & $-3.44 \mathrm{eV}$ & $-1.68 \mathrm{eV}$ & $1 f$ & $1 d$ \\
Basis 2 & $-3.35 \mathrm{eV}$ & $-1.42 \mathrm{eV}$ & $2 f$ & $2 d 1 f$ \\
Basis 3 & $-3.08 \mathrm{eV}$ & $-0.84 \mathrm{eV}$ & $3 f 2 g$ & $3 d 2 f 1 g$ \\
\hline \hline
\end{tabular}

With complete neglect of dynamic correlation (CASSCF), the HS is by $3.08 \mathrm{eV}$ lower in energy than the LS.

In the MCCEPA calculations only the Fe $3 d$ orbitals and the lone pairs of the ligands which point to iron were included in the correlation treatment. The HS-LS difference was reduced to $0.84 \mathrm{eV}$, but the results were still not converged with the number of correlated orbitals and the size of the basis set. A summary of our results is listed in Table II. Notice that it is actually well known that due to approximations in the treatment of dynamical correlations, multireference correlation methods cannot easily shed additional light onto this particular ground state problem. ${ }^{46,47}$

\section{Effective spin Hamiltonian}

In the HS $Q=0$ system a bunch of energetically low-lying electronic states arises from the spin coupling of the local spin $(S=2)$ at the Fe center and the ligand electrons. All these low-lying electronic states were investigated by CASSCF. (The basis set in these calculations was obtained from basis 1 of Table II by adding a semidiffuse $d$ function at $\mathrm{Fe}$ and removing the $f$ function at $\mathrm{Fe}$.) The exchange coupling constant for the antiferromagnetic coupling can be extracted from these calculations.

The active space contained the five $\mathrm{Fe} 3 d$ orbitals and the two singly occupied ligand orbitals with eight electrons. Twelve energetically low-lying electronic states were observed and the orbitals were optimized for a state average of these states: three septet states, six quintet states, and three triplet states. The results are summarized in Table III. The energy splitting of the lowest four states arises from the coupling of the $\mathrm{Fe}^{2+}$ ground state with the two ligand electrons, which can be described by a Heisenberg-Hamiltonian of the form

$$
H=-2 J \mathbf{S}_{\mathrm{Fe}} \mathbf{S}_{\mathrm{L} 1}-2 J \mathbf{S}_{\mathrm{Fe}} \mathbf{S}_{\mathrm{L} 2}-2 J_{\mathrm{LL}} \mathbf{S}_{\mathrm{L} 1} \mathbf{S}_{\mathrm{L} 2},
$$

where $J$ is the magnetic exchange coupling of a ligand electron with the Fe center, $J_{\mathrm{LL}}$ the coupling of the two ligand electrons with each other. The eigenvalues of the states can be obtained by diagonalizing the Heisenberg-Hamiltonian and are given in terms of the magnetic exchange coupling constants $J$ and $J_{\mathrm{LL}}$ in Table III. With these expressions we obtained the values $J=-21.4 \mathrm{meV}$ and $J_{\mathrm{LL}} \approx-0.18 \mathrm{meV}$ from the CASSCF energies.

The next eight states refer to the coupling of the low-lying excited ${ }^{5} E$ state at the $\mathrm{Fe}^{2+}$ center with the ligand electrons. The observed splitting does not obey the simple Heisenberg
TABLE III. CASSCF results for complex $1\left[\mathrm{Fe}(\mathrm{bpp})_{2}\right]^{2+}, Q=0$, in HS configuration. The first four states arise from different spin couplings of the $\mathrm{Fe}^{2+}$ ground state with the ligand electrons, the next states refer to the coupling of the low-lying excited $E$ state at the $\mathrm{Fe}^{2+}$ center with the ligand electrons.

\begin{tabular}{lccc}
\hline \hline State & Energy $(\mathrm{meV})$ & $E\left(J, J_{\mathrm{LL}}\right)$ & $J(\mathrm{meV})$ \\
\hline${ }^{3} B_{1}$ & 0.0 & $6 J-1 / 2 J_{\mathrm{LL}}$ & \\
${ }^{5} B_{1}$ & 87.0 & $2 J-1 / 2 J_{\mathrm{LL}}$ & -21.8 \\
${ }^{5} A_{1}$ & 126.9 & $3 / 2 J_{\mathrm{LL}}$ & $J_{\mathrm{LL}} \approx-0.16$ \\
${ }^{7} B_{1}$ & 210.4 & $-4 J-1 / 2 J_{\mathrm{LL}}$ & -21.0 \\
${ }^{5} B_{2} /{ }^{5} B_{3}$ & 261.1 & & \\
${ }^{3} B_{2} /{ }^{3} B_{3}$ & 318.2 & & \\
${ }^{7} B_{2} /{ }^{7} B_{3}$ & 364.5 & & \\
${ }^{5} B_{2} /{ }^{5} B_{3}$ & 419.7 & & \\
\hline \hline
\end{tabular}

picture due to an interaction of the quintet states of the same symmetry. In the given orbital space, the next states follow at energies of about $1.0 \mathrm{eV}$ above the ground state.

Summarizing, from the analysis of this spin Hamiltonian one thus expects a triplet ground state ${ }^{3} B_{1}$ separated by at least $90 \mathrm{meV}$ from the next electronic state $\left({ }^{5} B_{1}\right)$.

\section{Zero field splitting of the triplet ground state}

Spin-orbit coupling was considered using the scaled nucleus approximation in the same way as in our former work on Co complexes. ${ }^{48}$ A review on the applicability of approximate spin-orbit operators has been given by Neese. ${ }^{49}$ In the subspace of the CASSCF states which arise from the coupling of the two ligand electrons to the five $\mathrm{Fe}$ (II) quintet states of the $3 d^{6}$ configuration a spin-orbit configuration interaction calculation (SOC-CI) was performed. In the scaled nucleus approximation only the one electron spin-orbit integrals are calculated. To take into account the effects of the two electron spin spin and spin other orbit integrals the one electron integrals are scaled in such a way that a SOC-CI for the ${ }^{5} D$ ground state of the free $\mathrm{Fe}(\mathrm{II})$ reproduces the experimental spin-orbit splitting. ${ }^{50}$ A scaling parameter of 0.55 was obtained. This value is close to the value of 0.535 which was obtained by Koseki et al. ${ }^{51}$ In our calculation on $[\mathrm{Fe}(\mathrm{bpp})]^{0}$ the triplet ground state was split into a degenerate ground state $\left(m_{s}= \pm 1\right)$ and an excited state at $0.45 \mathrm{meV}$ higher energy.

\section{TRANSPORT EXPERIMENTS}

Our theoretical investigations suggest that the spin transition should manifest itself in an electron transport experiment by the specific structure of its magnetic excitations. We consider a three-terminal device (Fig. 5) where the molecule together with additional anchoring groups forms a molecular wire with a charge $Q$ which is controlled by the gate potential. Inelastic tunneling spectroscopy can be used to probe low-energy excitations in a particular charge state, which manifest themselves as resonances in the differential conductance $d I / d V$ at bias voltages $\delta E$. If the molecular coupling to the electrodes is large enough, so that the Kondo temperature is comparable to the splitting $\left(T_{\mathrm{K}} \gtrsim \delta E\right)$, then a nonequilibrium Kondo effect (i.e., a split Kondo peak) can develop. ${ }^{52}$ 
(a)

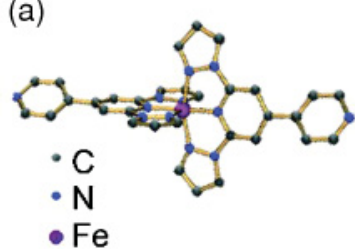

(b)

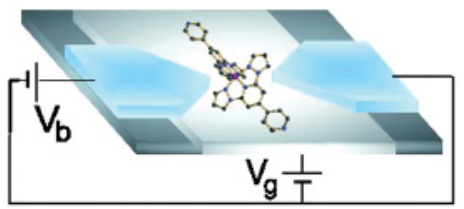

FIG. 5. (Color online) Left: The spin transition complex together with the linker units that were employed in the experimental measurements: $\left.\left[\mathrm{Fe}-(\mathrm{L})_{2}\right]^{2+}\right]$ complex $\left[L=4^{\prime}-\left(4^{\prime \prime \prime}-\right.\right.$ pyridyl $)-1,2^{\prime}$ : $6^{\prime} 1^{\prime \prime}$-bis-(pyrazolyl)pyridine]. Right: Schematic representation of the three-terminal molecular junction setup.

To connect the molecule, we fabricate nanometer sized gaps by a controlled electromigration process on a thin Au wire deposited on top of an oxidized Al gate. ${ }^{53,54}$ Electromigration and the subsequent self breaking of the wire is carried out at room temperature in a solution containing the molecule; the solvent is subsequently evaporated. If a molecule is trapped in the gap, a three-terminal device is formed in which the molecule connects via tunnel barriers to source and drain electrodes and couples electrostatically to an external gate [see Fig. 5(b)].

\section{A. Measurement results}

We present in Figs. 6(a) and 7(a) the differential conductance versus source-drain $\left(V_{\mathrm{b}}\right)$ and gate $\left(V_{\mathrm{g}}\right)$ voltages obtained at $T=1.5 \mathrm{~K}$ for two samples $\mathrm{A}$ and $\mathrm{B}$. The (a)

(b)
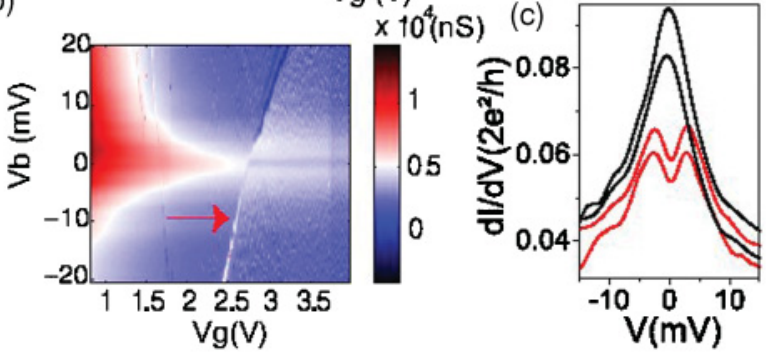

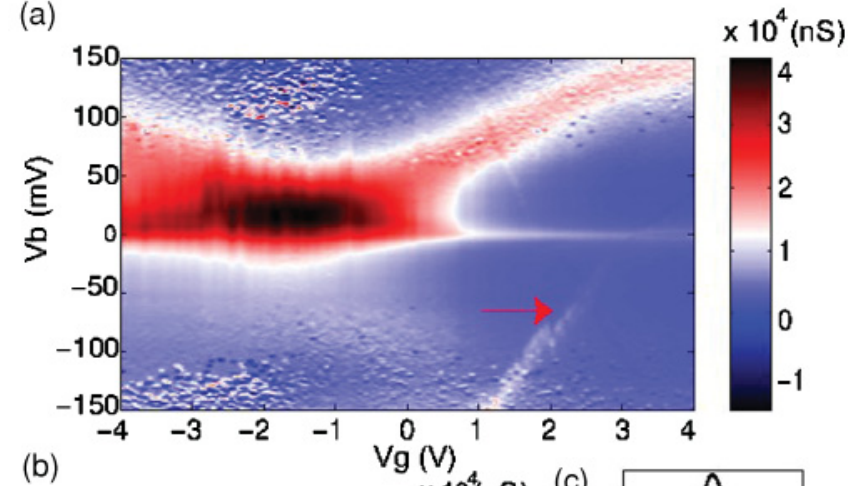

FIG. 6. (Color online) (a) Differential conductance of sample A vs source-drain $\left(V_{\mathrm{b}}\right)$ and gate $\left(V_{\mathrm{g}}\right)$ voltages. (b) Zoom of the differential conductance plot. The red (gray) arrows indicate the diagonal line discussed as an electrostatic shift in Sec. III. (c) Differential conductance traces (from top to bottom) vs $V_{\mathrm{b}}$ taken at gates voltages of, respectively, 2, 2.4, 2.9, and 3.3 V. The black (red/gray) traces correspond to gates voltages on the left (right) hand side of the diagonal line. data exhibits classical behavior of single-electron transport through a nano-object with a single relevant energy level. The extracted addition energies $E_{\text {add }}>100 \mathrm{meV}$ and gate couplings $\beta=C_{\mathrm{g}} / C_{\mathrm{tot}} \approx 0.02$ ( $C_{\mathrm{g}}$ : gate-dot capacitance; $C_{\mathrm{tot}}$ : total capacitance of the dot) are typical of transport through a single molecule. ${ }^{54}$ The tunnel couplings for samples A and B can be estimated from the Lorentzian broadening $\Gamma$ of the Coulomb peaks: $\Gamma_{\mathrm{A}} \approx 60 \mathrm{meV}$ and $\Gamma_{\mathrm{B}} \approx 10 \mathrm{meV}$.

Electrostatic shift. In addition to this typical singlemolecule transistor behavior we observe a particular feature visible as a diagonal line (indicated by a red/gray arrow) in the differential conductance plots of Figs. 6(a) and 6(b) and 7(b) and 7(c). In Fig. 7(b) on the right hand side of this line, a shift $\Delta V_{\mathrm{g}}$ of the Coulomb diamond edges in gate voltage

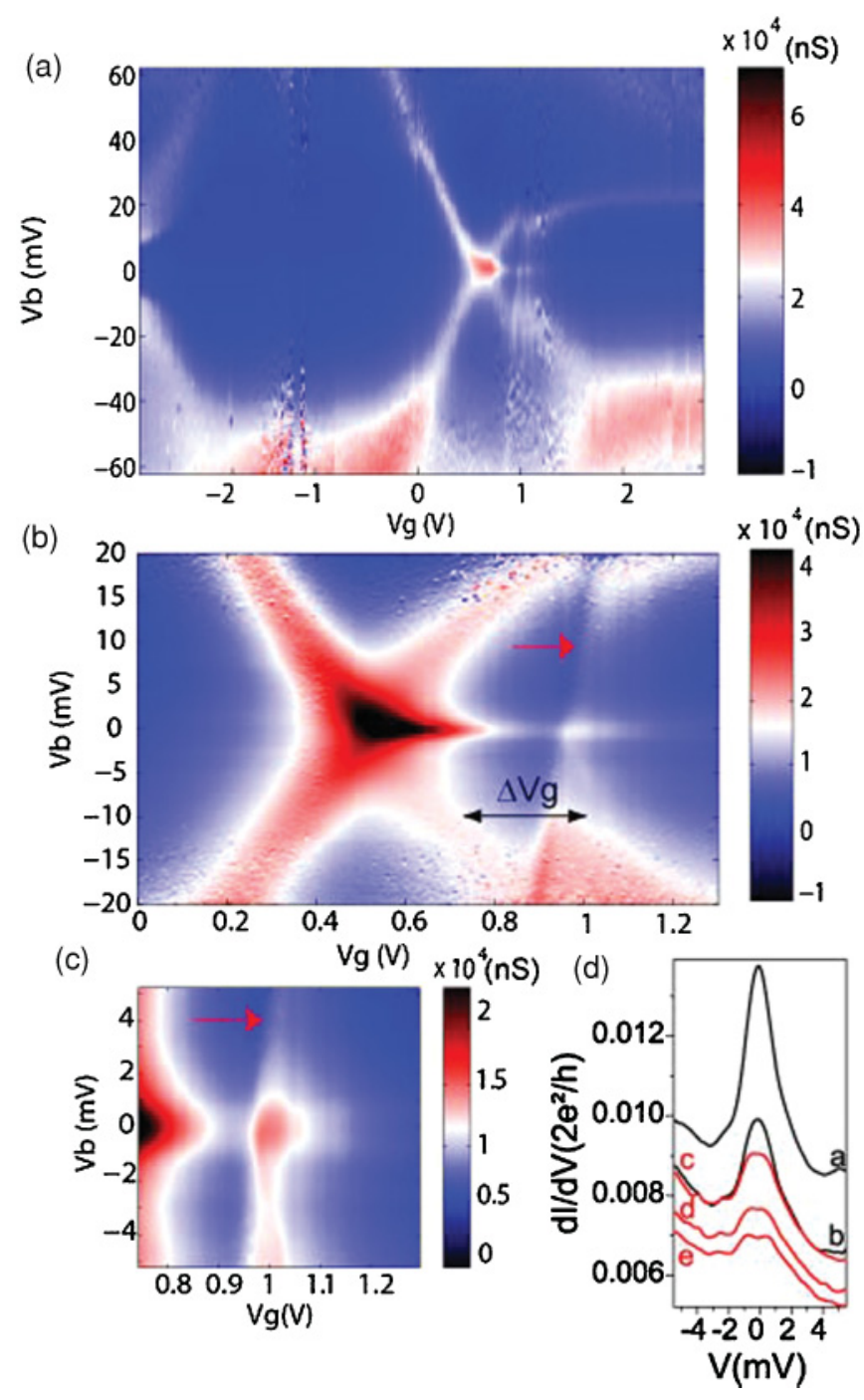

FIG. 7. (Color online) (a) Differential conductance of sample B vs source-drain $\left(V_{\mathrm{b}}\right)$ and gate $\left(V_{\mathrm{g}}\right)$ voltages. (b) and (c) Zoom of the differential conductance plot. The red (gray) arrows indicate the diagonal line discussed as an electrostatic shift in Sec. III. (d) Differential conductance traces (from a to e) vs $V_{\mathrm{b}}$ taken at gate voltages of, respectively, $0.8,0.9,1.15,1.2$, and $1.25 \mathrm{~V}$. The black (red/gray) traces correspond to gates voltages on the left (right) hand side of the diagonal line. From the full width at half height of the trace a we estimate a Kondo temperature $T_{\mathrm{K}}^{\mathrm{B}} \approx 25 \mathrm{~K}$. 
is present, which finds a natural interpretation as a response to a modification of the electrostatic environment, see below Sec. IV A.

Kondo peak. We now focus on the low-bias regime. A zoom of this region is shown in Figs. 6(b) and 7(c) as well as differential conductance traces versus $V_{\mathrm{b}}$ taken for different gate voltages in Figs. 6(c) and 7(d). On the left hand side of Figs. 6(b) and 7(c), a zero-bias peak (ZBP), characteristic of the Kondo effect, is observed. As the gate voltage is increased, one can first see a decrease of the ZBP height which is due to the reduction of the Kondo temperature as we go away from the degeneracy point. ${ }^{55}$ For sample A the temperature dependence of the Kondo peak and the zerobias conductance $G_{0}(T)$ at a given gate voltage are represented in Figs. 8(a) and 8(c). The zero-bias conductance temperature dependence $G_{0}(T)$ is compared to numerical renormalization group (NRG) predictions for $S=1 / 2$ and $S=1$ Kondo models using analytical fitting functions of the form $G_{S}(T)=G(0) f_{S}\left(T / T_{\mathrm{K}}\right)^{29}$ to which we add a constant term corresponding to background conductance $G_{\mathrm{bg}} \cdot{ }^{56} G_{0}(T)$ is in good agreement with $S=1 / 2$ Kondo effect for $G_{\mathrm{bg}}=$ $0.025 e^{2} / h, G(0)=0.061 e^{2} / h$, and $T_{\mathrm{K}}^{\mathrm{A}}=56 \mathrm{~K}$ suggesting a $S=1 / 2$ spin state for the molecule on the left hand side of Fig. 6(b).

Split Kondo peak. On the right hand side of Fig. 6(b) the ZBP is clearly split in two peaks $\pm e V_{\mathrm{b}}= \pm \Delta_{\mathrm{A}}= \pm 2.75 \mathrm{meV}$. The zero-bias conductance of the split ZBP $G_{0}(T)$ represented in Fig. 9(c) exhibits a nonmonotonic temperature dependence. For sample B a split ZBP is also observed on the right hand side of Fig. 7(c). From the trace indicated as "e" in Fig. 7(d) we extract a splitting $\Delta_{\mathrm{B}} \approx 0.5 \mathrm{meV}$. In this case, the splitting is less pronounced due to a smaller ratio $\Delta_{\mathrm{B}} / T_{\mathrm{K}}^{\mathrm{B}}$ compared to sample A. The splitting is however visible as a reduction of the zero-bias conductance if one compares the traces indicated as "b" and "c" in Fig. 7(d). When the gate voltage is further increased the splitting becomes more pronounced due to the decrease of the Kondo temperature as we go away from the degeneracy point. ${ }^{55}$

\section{THEORETICAL ANALYSIS OF THE EXPERIMENT}

\section{A. Double-dot system}

The electrostatic shift that gives rise to the diagonal lines in Figs. 6 and 7 can be reproduced by a Coulomb blockade model which is based on a scenario with two parallel quantum dots: current flows through one QD, the "transfer dot", $\mathrm{T}$ in the presence of a second dot, the "spectator dot" S [see Fig. 9(b)]. This second dot is electrostatically coupled to the first dot and as its tunnel coupling to one of the electrodes is very weak there is no net current through it. However, when the gate voltage is increased this dot can be charged by an additional electron, thereby, modifying the electrostatic environment of the other dot via their mutual capacitance $C_{\mathrm{M}}$. This charging induces an horizontal shift of the Coulomb diamond edges by $\Delta V_{\mathrm{g}}$. A similar effect realized on a larger scale with $\mathrm{C}_{60}$ molecules near nanotubes has been investigated recently. ${ }^{57}$ Figure 9(a) shows the result of a rate equation calculation of two single-level QDs capacitively coupled (see Appendix B

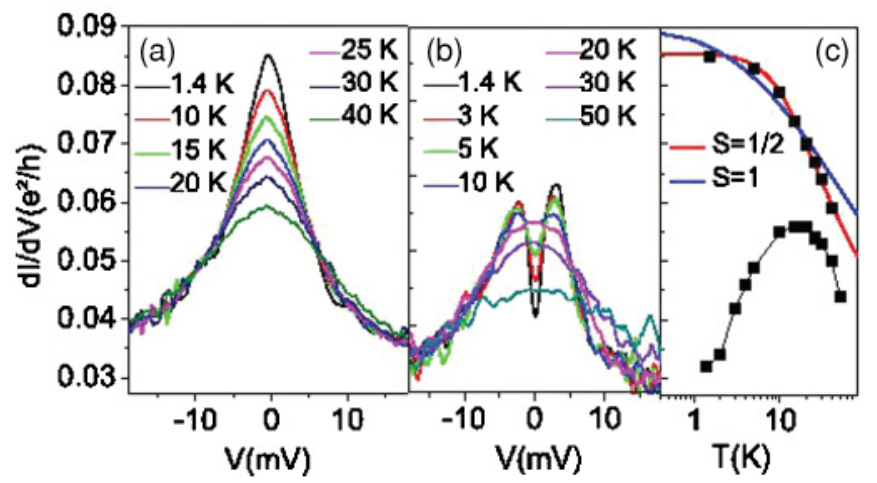

FIG. 8. (Color online) (a) and (b) Temperature dependence of differential conductance traces of sample A vs $V_{\mathrm{b}}$ taken, respectively, for $V_{\mathrm{g}}=2.2 \mathrm{~V}$ and $V_{\mathrm{g}}=3.2 \mathrm{~V}$. (c) Temperature dependence of the zero bias conductance in (a) and (b). The red (light gray) and blue (dark gray) lines represent the fits obtained from the analytical fitting functions ${ }^{29}$ of the form $G_{S}(T)=G(0) f_{S}\left(T / T_{\mathrm{K}}\right)+G_{\mathrm{bg}}$. For $S=1 / 2$ the parameters are $G(0)=0.061 e^{2} / h, T_{\mathrm{K}}=56 \mathrm{~K}$, and for $S=1$ they are $G(0)=0.064 e^{2} / h, T_{\mathrm{K}}=73 \mathrm{~K}$, with $G_{\mathrm{bg}}=0.025 e^{2} / h$ for both fits.

for the model parameters), which mimics the situation for sample B.

We discuss the physical origin of the observed double-dot behavior. Two principally different realizations of this doubledot system are conceivable. In one scenario the spectator dot is not part of the molecule. Summarizing the arguments presented in Appendix $\mathrm{C}$ we believe that this scenario is unlikely because the exchange coupling between the external spectator dot and the transfer dot (molecule) is too small, in general, in order to explain the observed zero-bias peak splitting. By contrast the double-dot behavior finds a natural realization just in the molecule $\left[\mathrm{Fe}(\mathrm{bpp})_{2}\right]^{2+}$ itself due to its internal

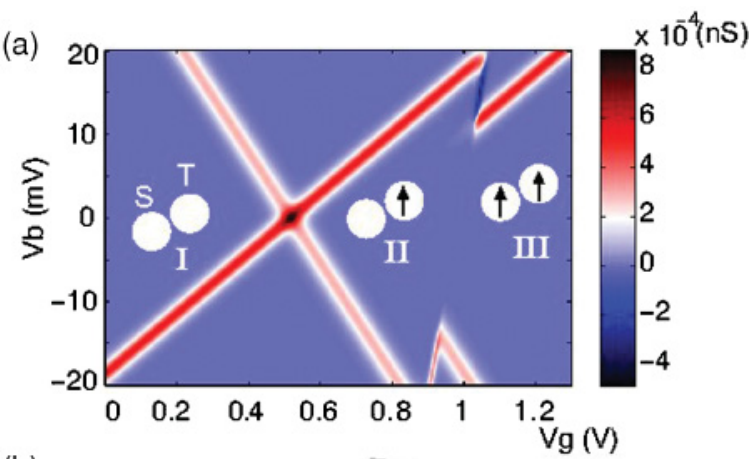

(b)

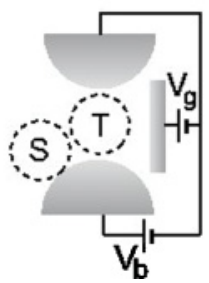

(c)

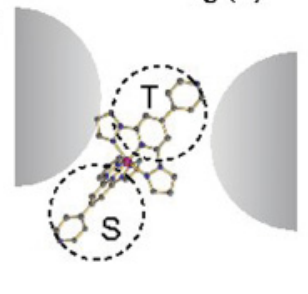

FIG. 9. (Color online) (a) Simulation of the differential conductance vs source-drain $\left(V_{\mathrm{b}}\right)$ and gate $\left(V_{\mathrm{g}}\right)$ voltages calculated for $T=4.2 \mathrm{~K}$. (b) Schematic representation of the double-dot system. T: transfer dot, S: spectator dot. (c) Schematic representation of molecule-electrode contacts realizing the double-dot system. 

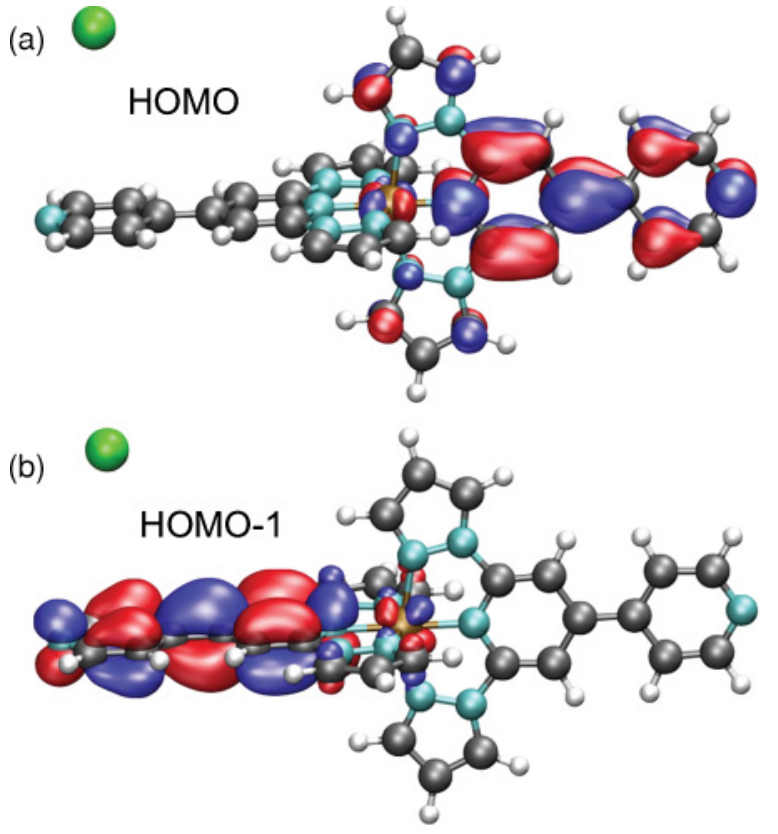

FIG. 10. (Color online) Two highest occupied molecular orbitals (HOMO-1 and HOMO) of $\left[\mathrm{Fe}^{\mathrm{II}}(\mathrm{L})_{2}^{-}\right]^{0}$. A green (light gray) dot represents a positive, point-like countercharge, $5.29 \AA$ above the outermost pyridine ring.

molecular structure. To see this we summarize the results from the previous theory section: The two bi-pyrazolyl-pyridine ligands support $\pi$ systems which are oriented perpendicularly to each other by the octahedral coordination around the $\mathrm{Fe}^{2+}$ metal ion. Therefore, left and right ligand states hybridize only weakly with each other. In particular, for the situation of double reduction (i.e., when two excess electrons are added) our calculations confirm that each electron is localized on one ligand only, with very little excess charge penetrating the region connecting the two ligands near the $\mathrm{Fe}^{2+}$ ion.

To further illustrate this important point we represent in Figs. 10(a) and 10(b) the HOMO and HOMO-1 of the molecule; these orbitals carry the additional electrons (double reduction). In the presence of a symmetry breaking potentialhere a countercharge (Fig. 10, green/gray ball)—each electron is located on its own ligand. This finding is generic, as may be inferred from the fact that the picture remains effectively unchanged when the separation of the countercharge to the molecule is modified (3.7-6.35 Å range has been tested).

In the asymmetric situation, where one ligand-the "transfer ligand" - is connected with two electrodes via $\pi$-ligand mediated interactions and the other one- the "spectator ligand"only to a single electrode [as represented in Fig. 9(c)], the double-dot system sketched in Fig. 9(b) is indeed realized.

This explanation is consistent with the observed transport characteristics in Figs. 6(a) and 7(b), if we assume the occupancy of the ligands to be as represented schematically in Fig. 9(a). On the left hand side (region I) the transfer ligand has an even occupancy [as confirmed by the absence of Kondo peak in this region in Fig. 7(b)]. When the gate voltage is increased (region II), the transfer ligand is charged by an additional electron, leading to an odd occupancy, $S_{t}=1 / 2$ and the observation of a Kondo peak [Figs. 6(a) and 7(b)].
In region III the spectator ligand is in turn charged leading to an odd occupancy $S_{t}=S_{s}=1 / 2$ of both dots, if an even occupancy is assumed for the spectator dot on the left hand side of the diagram. The analysis of the presence of a split Kondo peak in that region is discussed in the next paragraph.

\section{B. Origin of the split Kondo peak in region III}

Split ZBP as well as nonmonotonic temperature dependence of $G_{0}(T)$ are reminiscent of spin-spin interactions competing with Kondo screening, as observed in coupled QDs systems ${ }^{58,59}$ and described theoretically. ${ }^{60,61}$ In the case of our molecule, such interaction between two $S=1 / 2$ (one on each ligand) would be too weak to explain the observed splitting of the order of few milli-electron-volt (see Appendix C).

This analysis, however, is based on the assumption that the molecule is still in the LS state in region III. Our detailed theoretical analysis from Sec. II on the other hand suggests that the LS state may give way against a HS state when the $\left[\mathrm{Fe}(\mathrm{bpp})_{2}\right]^{2+}$ molecule is doubly charged. Accordingly, the molecule could be in its LS state in region I and II, while it would be in the HS state in region III. Recall, that in HS the $\mathrm{Fe}^{2+}$ core possesses a spin $S=2$ which is antiferromagnetically coupled to the spins on the ligands. The ground state is thus a spin triplet. ${ }^{63}$ Due to the spin-orbit interaction the triplet splits into a Kramer's doublet and a singlet state. In the optimized high-spin geometry of our molecule, we find that this zero field splitting $\delta E$ is about $0.45 \mathrm{meV}$. We show in Appendix D for $\mathrm{Fe}(\mathrm{L})_{2}$ that this value will be larger if distortions due to incorporation in the planar device geometry are present. Our calculations suggest that it can reach values of several milli-electron-volt. Such spin-orbit split $S=1$ states could explain our observation of split Kondo peaks with milli-electron-volt range spacing. Similar $S=1$ split Kondo peaks were also reported in transport measurements through a Co complex. ${ }^{29}$

\section{CONCLUSIONS}

In conclusion, we presented a detailed analysis of a new phenomenon - a spin transition controlled by an electrical field. We have presented a theoretical analysis for an $\mathrm{Fe}^{\mathrm{II}}(\mathrm{L})_{2}$ spin transition complex based on the density functional theory that predicts the existence of the effect for molecules in the gas phase. According to this theory one could expect a zero bias anomaly, the spin split Kondo peak, as a manifestation of this transition if the singlet-triplet excitation gap is in energy comparable to the Kondo scale. Transport experiments in a molecular three-terminal geometry performed on this $\mathrm{Fe}^{\mathrm{II}}(\mathrm{L})_{2}$ species indeed reveal a double-quantum-dot behavior in combination with a split Kondo peak. We discuss a possible intramolecular origin of the double-dot system and argue that the charge-induced spin transition would indeed permit us to account for the observed split Kondo peak.

To reach a definitive conclusion in favor of this scenario, further experiments, which could involve, for example, a detailed measurement of the Kondo splitting in magnetic fields, would be needed. The observation of such a spin transition would be an important step toward the development of molecular spintronics, ${ }^{65}$ as it demonstrates the possibility 
of addressing and controlling with an electric field individual spin states engineered at the molecular scale.

\section{ACKNOWLEDGMENTS}

We are grateful to F. Schramm and P. Wölfle for many fruitful discussions on the present and related subjects. Also, K. F. and F. E. thank the Center of Functional Nanostructures and (F.E., M.R.) the Priority Program 1243 of the Deutsche Forschungsgemeinschaft (DFG) as well as the HelmholtzGemeinschaft (project "Integrated Molecular Switches") and the Dutch funding agency FOM for support.

\section{APPENDIX A: ELECTRONIC STRUCTURE OF SPIN-TRANSITION COMPLEXES IN THE GAS PHASE}

\section{DFT results for the $\left[\mathrm{Fe}(\mathrm{bpp})_{2}\right]^{2+}$ complex}

So far our discussion of the ST in $\left[\mathrm{Fe}(\mathrm{bpp})_{2}\right]^{2+}$ was in terms of ground state properties: total energies, charge, and spin densities. We now briefly touch upon the reorganization of the core electronic structure. The associated spectral properties displayed in Fig. 11 offer further insight into the interplay between geometric and electronic degrees of freedom.

$L S$ spectrum. For LS and $Q=+2$ it is expected that the octahedral symmetry of the organic cage splits the core based $d$ orbitals into three nonbonding (lower) levels $\left(t_{2 g}\right)$ and two antibonding (upper) levels $\left(e_{g}\right)$ (Fig. 1). The (average) splitting between these states $\left(\Delta_{\text {oct }}\right)$ measures an energy for charge neutral excitations. Indeed, our LS data (Fig. 11, left) fully confirm this expectation. The corresponding numerical values for $\Delta_{\text {oct }}$ are given in Table I. One aspect of the schematics (Fig. 11) is particularly noteworthy. The octahedral splitting is so large that the $e_{g}$ levels of $\mathrm{Fe}^{\mathrm{II}}$ and the weakly hybridizing $\pi$-type LUMOs of the ligands cross each other. Therefore, in the absence of a magnetic transition, one expects an excess electron to localize on the ligands. ${ }^{66}$

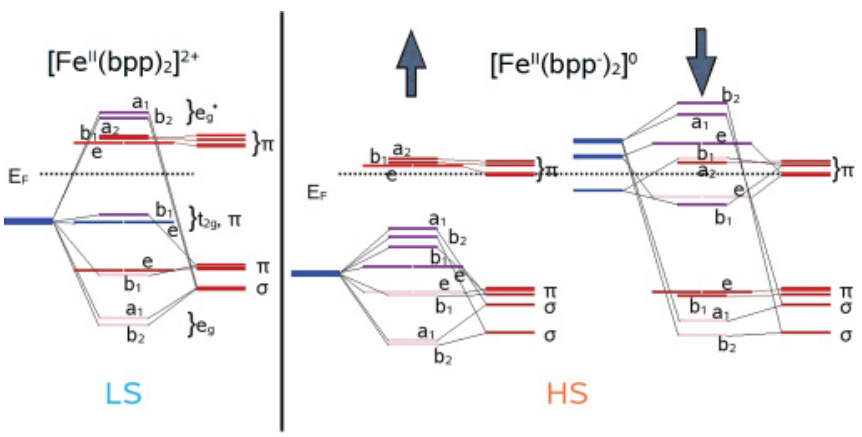

FIG. 11. (Color online) Genealogy of the Kohn-Sham orbitals from DFT. Left column of a term scheme indicates five degenerate $3 d$ orbitals of the free $\mathrm{Fe}(\mathrm{II})$ atom. Center column displays spectra of $\left[\mathrm{Fe}(\mathrm{bpp})_{2}\right]^{2+}$ (left set) and $\left[\mathrm{Fe}(\mathrm{bpp})_{2}^{-}\right]^{0}$ (two right sets). Right column indicates the frontier states of the complex $\left[\mathrm{Fe}(\mathrm{bpp})_{2}\right]^{Q}$ after the replacement $\mathrm{Fe}(\mathrm{II}) \rightarrow \mathrm{Mg}$ (II) serving as the reference state for the ligand orbitals. The relative energies in a given term scheme, center and right column, have been obtained with the TPSSH functional. Colors: red (gray)/pink (light gray) orbitals are preferentially located on the ligands, blue (dark gray)/magenta (gray) orbitals on the metal core. Chemical potential $\left(E_{F}\right)$ is placed in the middle of the HOMOLUMO gap.
$H S$ spectrum. The spectrum of the HS configuration $Q=$ 0 is remarkably different from the LS case $Q=+2$. Most striking in Fig. 11 on the right hand side is the overall splitting $\Delta$ of the Fe-based $d$ states. It is weaker by about a factor of 2 in HS (majority spin component $\Delta_{\mathrm{HS}} \approx 2 \mathrm{eV}$, TPSSH) as compared to LS ( $\Delta_{\mathrm{LS}} \approx 4.5 \mathrm{eV}$, TPSSH). As discussed before, $\Delta$ measures how strongly the ligand cage, specifically their $\mathrm{N}$ atoms, discriminate the five $3 d$-core orbitals. Because of the increased core size in the HS state, this interaction is much smaller than in LS.

When undergoing the ST, the ligand cage bends and shifts outward (see Fig. 3). In this process the octahedral symmetry cannot be maintained. Therefore we observe in Fig. 11 that the intramultiplet splitting $e_{g}$ and $t_{2 g}$ increases when comparing LS with the HS majority-spin term scheme, while the intermultiplet splitting decreases.

In the HS state $\Delta$ is diminished to such an extent that Hund's rule can be applied in order to fill orbitals: the five majority-spin orbitals fill up before the first minority one does (see Fig. 11). Importantly, due to the Coulomb interaction with the majority-spin electrons, the minority states with $3 d$ character are shifted upward in energy by an amount $U \approx$ $3 \mathrm{eV}$. This energy is so large that only one out of the five $3 d$ orbitals remains in energy below the ligand based $\pi$ orbitals, which do not feel the $U$ shift. As a consequence, HOMO and HOMO- 1 states in the $Q=0$ HS configuration are located on the ligands and carry minority spin electrons. We thus confirm the previous analysis: the two additional electrons do not flow into the Fe core but reside on the ligands only; they exhibit an antiferromagnetic pairing with the magnetic Fe(II) ion.

\section{Comparative study: $\mathrm{Fe}(\mathrm{tpy})$ and $\mathrm{Ru}(\mathrm{bpp})$}

We now continue our discussion with the complex 2 $\left[\mathrm{Fe}(\mathrm{tpy})_{2}\right]^{2+}$. The analysis proceeds along the same lines as for complex 1. From the energy differences $\Delta E$ given in Table IV one sees that also for complex 2 the HS state becomes increasingly favorable when charging the molecule. A clear transition is not observed reliably (i.e., for all $\mathrm{XC}$ functionals used) for $Q \gtrsim 0$, yet.

As a second reference, we introduce complex $\mathbf{3}$ $\left(\left[\mathrm{Ru}(\mathrm{bpp})_{2}\right]^{2+}\right)$. This system is a classic example in the present context because it has a relatively large splitting $\Delta_{\text {oct }}$ (see Tables V and VI). Indeed, the HS state, which is metastable at $Q=+2$ for complexes $\mathbf{1}, \mathbf{2}$, turns unstable for complex $\mathbf{3}$ in our calculations and it remains unstable also for $Q=0$, so here the ST is strongly suppressed.

TABLE IV. Complex $2\left[\mathrm{Fe}(\text { tpy })_{2}\right]^{2+}$ data analogous to Table I.

\begin{tabular}{|c|c|c|c|c|c|c|c|}
\hline \multirow[b]{2}{*}{$Q$} & \multirow[b]{2}{*}{$\mathrm{XC}$} & \multirow[b]{2}{*}{$\Delta E(\mathrm{eV})$} & \multicolumn{2}{|c|}{$\underline{\left[\mathrm{Fe}(\mathrm{tpy})_{2}\right]^{2+} \mathrm{HS}}$} & \multicolumn{3}{|c|}{$\left[\mathrm{Fe}(\mathrm{tpy})_{2}\right]^{2+} \mathrm{LS}$} \\
\hline & & & $d_{\mathrm{av}}^{\mathrm{HS}}(\AA)$ & $S(\mathrm{Fe})$ & $d_{\mathrm{av}}^{\mathrm{LS}}(\AA)$ & $S(\mathrm{Fe})$ & $\Delta_{\text {oct }}(\mathrm{eV})$ \\
\hline+2 & BP86 & 1.46 & 2.18 & 1.88 & 1.95 & 0.0 & 2.95 \\
\hline 0 & BP86 & 0.4 & 2.13 & 1.75 & 1.94 & 0.23 & 3.07 \\
\hline+2 & TPSSH & 0.79 & 2.19 & 1.91 & 1.96 & 0.00 & 4.05 \\
\hline 0 & TPSSH & 0.23 & 2.15 & 1.84 & 1.95 & 0.09 & 4.69 \\
\hline+2 & B3LYP & 0.09 & 2.22 & 1.88 & 2.0 & 0.0 & 5.80 \\
\hline 0 & B3LYP & -0.91 & 2.19 & 1.84 & 1.99 & 0.05 & 5.85 \\
\hline
\end{tabular}


TABLE V. Data for complex 3 [Ru(bpp $\left.)_{2}\right]^{2+}$, data analogous to Table I, column LS. Entries for HS are missing, since a metastable HS state could not be identified for this system.

\begin{tabular}{lcccc}
\hline \hline & & \multicolumn{3}{c}{$\left[\mathrm{Ru}(\mathrm{bpp})_{2}\right]^{2+} \mathrm{LS}$} \\
\cline { 3 - 5 }$Q$ & $\mathrm{XC}$ & $d_{\mathrm{av}}(\AA)$ & $S(\mathrm{Ru})$ & $\Delta_{\text {oct }}(\mathrm{eV})$ \\
\hline+2 & BP86 & 2.05 & 0.0 & 3.7 \\
0 & BP86 & 2.05 & 0.1 & 3.8 \\
+2 & TPSSH & 2.06 & 0.0 & 5.0 \\
0 & TPSSH & 2.06 & 0.05 & 5.0 \\
+2 & B3LYP & 2.08 & 0.0 & 5.58 \\
0 & B3LYP & 2.08 & 0.07 & 5.55 \\
\hline \hline
\end{tabular}

Note about elasticity and $\Delta_{\text {oct }}$. Summarizing our analysis, we arrive at the following physical picture: complex $\mathbf{1}$ is close to a magnetomechanical instability that is generated from the competition of the repulsive Coulomb interaction between the frontier state electrons and the ligand cage's elastic energy. Any manipulation that favors one of the two protagonists against the other can drive the transition. Changing the cage geometry, as has been done with $\mathbf{2}$, can discourage the core growth and thus a stronger stimulus is needed to flip into HS.

One might suspect that there should be a rule of thumb that says that the $\mathrm{M}(\mathrm{II})$ complex with the smaller excitation gap $\Delta_{\text {oct }}$ undergoes the ST more easily. However, our study shows that also the cage rigidity ("elasticity") is important, which decides about how difficult it is for the cage geometry to change. Elasticity in principle involves spectral properties not only of the highest occupied levels but also of the levels at much lower energies. Therefore, it is not just described by $\Delta_{\text {oct }}$ alone. However, we suggest here that the first property, the large gap $\Delta_{\text {oct }}$ in systems with (approximately) octahedral symmetry, may in fact be understood (at least in certain cases) as a measure of the second, namely cage rigidity.

The rule of thumb appears to be valid for the example of complex 2: it has a gap slightly bigger than the one of complex $\mathbf{1}$ and it is not as easily driven through the ST, indeed. In complex 3 the $\mathrm{Ru}(\mathrm{II})$ core with its $4 d$ shell is much bigger than the Fe(II) core with the $3 d$ shell. Therefore it fills the space within the ligand cage very tightly. The consequences are twofold.

(1) The symmetry breaking effect of the organic cage will be very strong for $\mathrm{Ru}(\mathrm{II})$ with a resulting splitting of $\Delta_{\text {oct }}$, that is relatively large. (Recall that the $\Delta_{\text {oct }}$ splitting between $e_{g}$ and $t_{2 g}$ levels essentially is a consequence of the broken rotational invariance of free space. ${ }^{73}$ ) Now, the rigid, narrow cage has a tendency to interfere with rotational invariance much more strongly than the squashy, big one. Therefore, strained, stiff systems tend to produce a bigger splitting $\Delta_{\text {oct }}$. We conclude that the validity of the empirical rule of thumb formulated above is closely related to the fact that the important cage elastic properties are implicitly taken into account.

(2) Due to the pressure of the $\mathrm{Ru}(\mathrm{II})$ core, the cage will be always under a strain much larger than for the reference systems 1, 2. Hence, the force necessary for Ru(II) to stretch the organic cage any further is much bigger than in the case of $\mathrm{Fe}$ (II). So, from this point of view the suppression of the magnetomechanical effects, which we observe, actually is expected.

\section{APPENDIX B: PARAMETRIZATION OF THE DOUBLE-DOT MODEL}

The diagonal line visible in the differential conductance plots of Figs. 6(a) and 6(b) and 7(b) and 7(c) can be understood by looking at a system consisting of two quantum dots coupled to a source, drain, and gate electrode. When the dots are noninteracting they both give rise to Coulomb diamonds, and the stability diagram is simply the sum of their conductances. However, when a capacitive interaction is present between the dots, switch lines can occur in the stability diagrams at the points where the diamond edges of the dots intersect.

In the case where one of the dots is much more weakly coupled than the other [a schematic representation is given in Fig. 9(b)], the switch line corresponds to where one of the diamond edges of the second dot would have been if they were observable. Since this dot is very asymmetrically coupled, the average charge only significantly changes near one of the diamond edges. Due to the capacitive interaction, the charging of the second dot results in an electrostatic potential change on the first dot, corresponding to an effective gate voltage jump at the diamond edge.

Figure 9(a) is the result of a rate equation calculation of two single-level quantum dots with a mutual capacitance. The coupling parameters have been fitted to the measurement in Fig. 7(b). The position of the diamond edge is determined by the chemical potential, which, for dot 1 , is given by

$$
\mu_{1}=\epsilon_{1}-e \alpha_{1} V_{b}-e \beta_{1} V_{g}+U
$$

where

$\alpha_{1}=\frac{C_{\mathrm{R} 1}-C_{\mathrm{L} 1}+\left(C_{\mathrm{R} 2}-C_{\mathrm{L} 2}\right) C_{\mathrm{M}} / C_{2}}{2 C_{1}-2 C_{\mathrm{M}}^{2} / C_{2}} \approx \frac{C_{\mathrm{R} 1}-C_{\mathrm{L} 1}}{2 C_{1}}$

TABLE VI. A comparison between theory and experiment: HOMO-LUMO gaps vs the lowest lying optical excitation, and the average Fe-N bond length $d_{\text {av }}$.

\begin{tabular}{|c|c|c|c|c|c|c|c|c|}
\hline \multirow[b]{2}{*}{ Complex } & \multicolumn{4}{|c|}{$d_{\mathrm{av}}(\AA)$} & \multicolumn{4}{|c|}{ H-L gaps (eV) } \\
\hline & BP86 & TPSSH & B3LYP & Expt. & BP86 & TPSSH & B3LYP & Expt. \\
\hline$\left[\mathrm{Fe}(\mathrm{bpp})_{2}\right]^{2+} \mathrm{HS}$ & 2.19 & 2.19 & 2.22 & $2.18^{67}$ & 0.31 & 2.12 & 3.22 & $1.29^{70}$ \\
\hline$\left[\mathrm{Fe}(\mathrm{bpp})_{2}\right]^{2+} \mathrm{LS}$ & 1.95 & 1.96 & 2.0 & $1.93^{68}$ & 2.05 & 3.15 & 4.08 & - \\
\hline$\left[\mathrm{Fe}(\text { tpy })_{2}\right]^{2+} \mathrm{LS}$ & 1.95 & 1.96 & 2.0 & $1.95^{69}$ & 1.86 & 2.84 & 3.67 & $2.3^{71}$ \\
\hline$\left[\mathrm{Ru}(\mathrm{bpp})_{2}\right]^{2+} \mathrm{LS}$ & 2.05 & 2.06 & 2.08 & - & 2.2 & 3.07 & 3.75 & $3.3^{72}$ \\
\hline
\end{tabular}


is the bias coupling (assuming the mutual capacitance $C_{\mathrm{M}}$ is much smaller than the total capacitances $C_{1}$ and $C_{2}$ of the dots),

$$
\beta_{1}=\frac{C_{\mathrm{G} 1}+C_{\mathrm{G} 2} C_{\mathrm{M}} / C_{2}}{C_{1}-C_{\mathrm{M}}^{2} / C_{2}} \approx \frac{C_{\mathrm{G} 1}}{C_{1}}
$$

is the gate coupling, and

$$
U=\frac{e^{2} C_{\mathrm{M}}}{C_{1} C_{2}-C_{\mathrm{M}}^{2}} \approx \frac{e^{2} C_{\mathrm{M}}}{C_{1} C_{2}}=\beta \Delta V_{\mathrm{G}}
$$

is the capacitive interaction energy. The parameters used in the simulation are $\epsilon_{1}=12 \mathrm{meV}, \alpha=0.147, \beta=0.023, \Gamma_{\mathrm{L}}=$ $\Gamma_{\mathrm{R}}=10 \mathrm{meV}$ for the dot $1, \epsilon_{2}=119 \mathrm{meV}, \beta=0.125, \Gamma_{\mathrm{L}}=$ $10 \mathrm{meV}, \Gamma_{\mathrm{R}}=10^{-3} \mathrm{meV}$, and $\Delta V_{\mathrm{G}}=0.2 \mathrm{~V}$. Since $\Gamma \gg k_{\mathrm{B}} T$ in the measurement, the linewidths are small in the calculation compared to the experiment because rate equation calculations do not take into account the broadening of the energy levels due to the coupling to the leads. The switch line, however, is clearly reproduced.

\section{APPENDIX C: DISCUSSION ON ALTERNATIVE SCENARIOS}

\section{Spectator dot outside the molecule}

As an alternative explanation to the one outlined in Sec. IV A, one could imagine an extrinsic spectator dot that does not form a chemical bond with the original molecule, for example, a second molecule or a small metallic grain (see Fig. 12).

In that case the direct tunneling between the spectator and the transfer dot should be expected to be smaller than in an intramolecular case since they are not connected via a chemical bond. Hence, also the associated exchange coupling will be very small. Of course, in principle there is an additional indirect interaction due to the RKKY coupling via the leads. An accurate estimation of this splitting is difficult to obtain as it depends strongly on the spatial configuration and geometry of the dots and electrodes. However, we argue that the associated exchange coupling should anyways be very weak. This is because coherent tunneling processes connecting the two dots

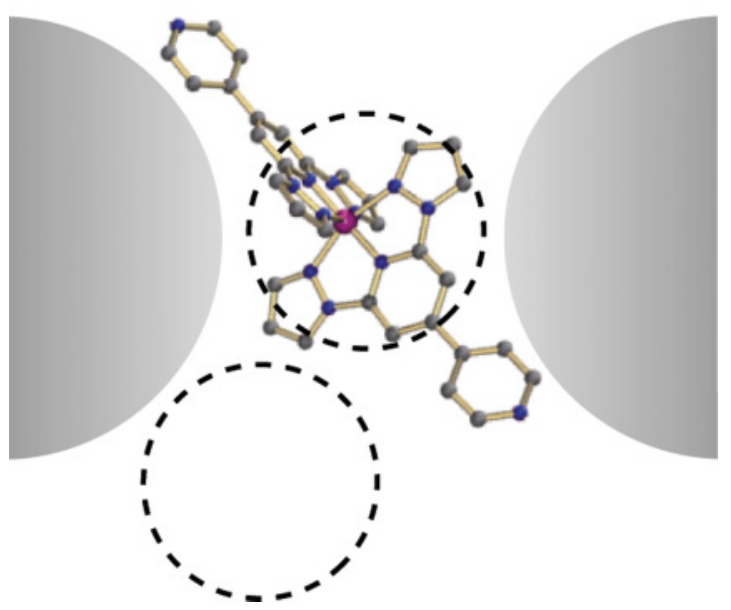

FIG. 12. (Color online) Schematic representation of the doubledot system in the external dot scenario. have to take electrons across two barriers: from the spectator dot into the leads and then from the leads into the transfer dot (and the same way back to establish coherence). The parametrical estimate ("golden rule" type) for the associated coupling is $J_{\mathrm{RKKY}}=\Gamma_{\mathrm{t}} \Gamma_{\mathrm{s}} / \Delta_{\mathrm{st}} /\left(r_{\mathrm{ts}} k_{\mathrm{F}}\right)^{2}\left(\Gamma_{\mathrm{t}, \mathrm{s}}\right.$ is the electrode induced broadening of the molecular levels of the transfer and spectator dot; $\Delta_{\mathrm{t}, \mathrm{s}}$ is the mismatch of the two coupled energy levels; $r_{\mathrm{ts}} k_{\mathrm{F}}$ is the spatial distance of the two contact points of the two dots with the electrodes measured in units of the Fermi wavelength). Assuming $\Gamma_{\mathrm{ts}} \approx 10 \mathrm{meV}, \Delta_{\mathrm{ts}} \approx 100 \mathrm{meV}$, and $r_{\mathrm{ts}} k_{\mathrm{F}} \approx 3-10$, typical values of $J_{\mathrm{RKKY}}$ lie between 0.01 and $0.1 \mathrm{meV}$. Hence we conclude an explanation of the observed split ZBP, which would be based on exchange interaction with an external dot seems hardly consistent with the values of the observed splittings $\Delta_{\mathrm{A}, \mathrm{B}}$.

\section{Double dot with two weakly coupled $S=1 / 2$ spins?}

We have also investigated to what extent the experimental findings may be understood in conventional terms of two weakly coupled quantum dots, each one carrying a spin $S=1 / 2$. As we shall see, such an explanation is not easily reconciled with combined experimental and theoretical evidence. As discussed in the paper our system is made of two capacitively interacting dots which we argue could be the two ligands of the molecule as represented in Fig. 9(c). Having this in mind one might expect that the split ZBP is induced by interactions between two spins, each on one ligand. This explanation would be consistent with the observed transport characteristics in Figs. 6 and 7, if we assume the occupancy of the ligands to be as represented schematically in Fig. 9(a). On the left hand side (region I) the transfer ligand has an even occupancy [as confirmed by the absence of Kondo peak in this region in Fig. 9(a)]. When the gate voltage is increased (region II), the transfer ligand is charged by an additional electron, leading to an odd occupancy $S_{\mathrm{t}}=1 / 2$ and the observation of a Kondo peak [Figs. 6(a) and 7(b)]. In region III the spectator ligand is in turn charged leading to an odd occupancy $S_{\mathrm{t}}=S_{\mathrm{s}}=1 / 2$ of both dots. A sizable spin interaction between the two ligands could then explain the experimentally observed peak splitting.

To check this scenario we determine the ligand-ligand exchange coupling (with the molecule being in the LS state). In Appendix D we provide a full fledged analysis of the magnetic excitations of the molecule in LS and HS state that is based on $a b$ initio calculations. They predict for the LS state a weak ligand-ligand ferromagnetic exchange coupling $J_{\mathrm{LS}}^{\mathrm{LL}} \approx 0.095 \mathrm{meV}$. Here we reproduce this result using a simple estimate $J_{\mathrm{LS}}^{\mathrm{LL}} \sim \varrho^{2} U\left(\varrho=t / E_{\mathrm{HOMO}^{*}}\right.$ is the relative energy splitting between symmetric/antisymmetric combinations of ligand orbitals; $U$ is the effective interaction energy in the near ion region of wave function overlap, roughly approximated by the $e_{g}-t_{2 g}$ splitting $U \lesssim \Delta_{\text {oct }}$ ). We have typically $\varrho \approx 0.1 \%-1 \%, U \approx 5 \mathrm{eV}$, so $J_{\mathrm{LS}}^{\widetilde{\mathrm{LL}}} \lesssim 0.01-1 \mathrm{meV}$. Essentially, this low value is due to the suppression of the tunneling matrix elements $t$ between the two ligand systems.

Our $a b$ initio analysis provides additional information. The exchange couplings are only weakly modified if a small variation of the $90^{\circ}$ angle between the ligands is taken into account $\left(0.15 \mathrm{meV}\right.$ change at $\left.20^{\circ}\right)$. Such deviations from the 
TABLE VII. Distances between the Fe center and nearest neighbor $\mathrm{N}$ sites (on and off long axis) for different XC functionals. All distances in Ångström.

\begin{tabular}{llllll}
\hline \hline & \multicolumn{2}{c}{ High Spin } & & \multicolumn{2}{c}{ Low Spin } \\
\cline { 2 - 3 } \cline { 5 - 6 } $\begin{array}{l}\text { XC } \\
\text { functional }\end{array}$ & $\begin{array}{l}\text { Fe-N dist. } \\
\text { on long axis }\end{array}$ & $\begin{array}{l}\text { Fe-N dist. } \\
\text { off axis }\end{array}$ & & $\begin{array}{l}\text { Fe-N dist. } \\
\text { on long axis }\end{array}$ & $\begin{array}{l}\text { Fe-N dist. } \\
\text { off-axis }\end{array}$ \\
\hline BP86 & 2.17 & 2.19 & & 1.90 & 1.97 \\
TPSSH & 2.17 & 2.21 & & 1.91 & 1.99 \\
B3LYP & 2.19 & 2.24 & & 1.93 & 2.03 \\
\hline \hline
\end{tabular}

geometry of the molecule optimized in the gas phase may occur due to the experimental boundary conditions set, for example, by the electrodes.

To further support our arguments, we compare the theoretical value with observed exchange splittings for LS transition metal complexes. They are indeed very small, in the micro-electron-volt range. ${ }^{62}$ In conclusion, according to our calculations, when assuming a LS state, the estimated ligand-ligand exchange coupling is too small to explain the observed splitting $\Delta_{\mathrm{A}, \mathrm{B}}=0.5-3 \mathrm{meV}$ in region III.

\section{APPENDIX D: MAGNETIC EXCITATIONS OF THE SPIN-TRANSITION COMPLEX $\left[\mathrm{Fe}(\mathrm{L})_{2}\right]^{2+}$}

\section{Structure optimization}

As in the previous sections, DFT calculations have been employed for structure optimization employing the TURBOMOLE machinery and a def-TZVP (triple- $\zeta$ valence plus polarization ${ }^{39,40}$ ) basis set was used along with default convergence criteria for the electronic convergence $\left(10^{-6}\right.$ hartree $)$ and geometry gradient $\left(10^{-3}\right.$ hartree/bohr). Again three different functionals have been compared: TPSSH, ${ }^{37} \mathrm{BP} 86,{ }^{36}$ and B3LYP ${ }^{38}$ As may be inferred from Table VII, the calculations for all three functionals were consistent. The DFT geometry optimized structures (TPSSH) were used as input structures in the wave function based calculations described further below.

\section{Low energy excitations}

The calculated data, that we presented so far, was obtained for $\left[\mathrm{Fe}(\mathrm{bpp})_{2}\right]$ while $\left[\mathrm{Fe}(\mathrm{L})_{2}\right]$ was used in the measurements. Here we analyze the effects of the pyridine anchor groups. The results on magnetic excitation energies and zero field splitting were obtained exactly with the same methods and basis sets as for $\left[\mathrm{Fe}(\mathrm{bpp})_{2}\right]$. In some calculations a countercharge $q=|e|$

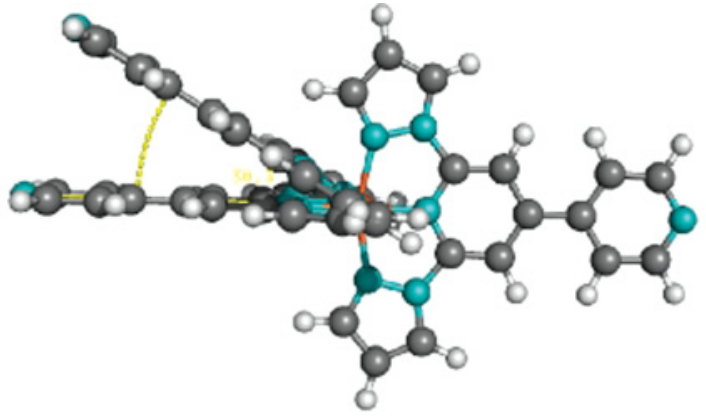

FIG. 13. (Color online) Easy axis tilting geometry used in order to estimate the impact of molecular deformations on total energies and exchange couplings (Table IX).

was included which was simulated by a frozen $\mathrm{Li}^{+}$ion in the CASSCF calculations. The $\mathrm{Li}^{+}$was equipped with a Huzinaga $7 s \rightarrow 1 s$ basis with contraction coefficients optimized for a free $\mathrm{Li}^{+}$ion to account for Pauli repulsion and thus to avoid artificial charge transfer.

Results for $L S$. The coupling is given by a simple Heisenberg Hamiltonian:

$$
H=-2 J_{\mathrm{LL}} \mathbf{S}_{\mathrm{L} 1} \mathbf{S}_{\mathrm{L} 2} \text {. }
$$

For $S_{\mathrm{L}}=1 / 2$ at each ligand a triplet and a singlet state with energy difference $E(S=1)-E(S=0)=-2 J_{\mathrm{LL}}$ are obtained in the CASSCF calculations.

The total energies and coupling constant for the triplet and singlet state of LS are listed in the third column of Table VIII. The two ligand electrons are almost uncoupled. Furthermore, we investigated whether the coupling is influenced by a change of the $90^{\circ}$ angle between the ligands (see Fig. 13). As may be inferred from Table IX, going from $0^{\circ}$ to $20^{\circ}$, the exchange coupling is increased by a factor of 2 , but it is still very small, while the energy cost of the tilting is already in the electron volt range.

Results for HS. The Heisenberg Hamiltonian is given by

$$
H=-2 J_{\mathrm{LL}} \mathbf{S}_{\mathrm{L} 1} \mathbf{S}_{\mathrm{L} 2}-2 J \mathbf{S}_{\mathrm{Fe}} \mathbf{S}_{\mathrm{L} 1}-2 J \mathbf{S}_{\mathrm{Fe}} \mathbf{S}_{\mathrm{L} 2},
$$

$J$ is the exchange coupling constant for the coupling between the Fe center with $S_{\mathrm{Fe}}=2$. Total energies and exchange coupling constants for $\mathrm{HS}$ are given in the fourth column of Table VIII. The coupling between the Fe center and the ligand electrons $J$ is two orders of magnitude larger than the ligand-ligand coupling $J_{\mathrm{LL}}$. Considering that the calculation of $J_{\mathrm{LL}}$ depends on differences of very

\begin{tabular}{|c|c|c|c|c|}
\hline & & LS & HS & HS with point charge \\
\hline$E(S=0)$ & & $-3139.505349 \mathrm{H}$ & & \\
\hline$E(S=1)$ & $6 J-1 / 2 J_{\mathrm{LL}}$ & $-3139.505356 \mathrm{H}$ & $-3139.644114 \mathrm{H}$ & $-3146.887888 \mathrm{H}$ \\
\hline$E(S=2, a)$ & $2 J-1 / 2 J_{\mathrm{LL}}$ & & $-3139.642004 \mathrm{H}$ & $-3146.886012 \mathrm{H}$ \\
\hline$E(S=2, b)$ & $3 / 2 J_{\mathrm{LL}}$ & & $-3139.641007 \mathrm{H}$ & $-3146.884903 \mathrm{H}$ \\
\hline$E(S=3)$ & $-4 J-1 / 2 J_{\mathrm{LL}}$ & & $-3139.638951 \mathrm{H}$ & $-3146.883076 \mathrm{H}$ \\
\hline$J$ & & 0 & $-13.9 \mathrm{meV}$ & $-13.3 \mathrm{meV}$ \\
\hline$J_{\mathrm{LL}}$ & & $0.095 \mathrm{meV}$ & $-0.08 \mathrm{meV}$ & $1.55 \mathrm{meV}$ \\
\hline
\end{tabular}

TABLE VIII. Low energy (spin) excitations of the $\left[\mathrm{Fe}^{\mathrm{II}}(\mathrm{L})_{2}\right]^{0}$ complex. 
TABLE IX. Increase of the ground-state energy of the molecule upon tilting in LS state. For the definition of the angle see Fig. 13.

\begin{tabular}{lcc}
\hline \hline Angle & $E_{\text {tilt }}(\mathrm{eV})$ & $J_{\mathrm{LL}}(\mathrm{meV})$ \\
\hline $0^{\circ}$ & 0.0 & 0.095 \\
$10^{\circ}$ & 0.117 & 0.122 \\
$20^{\circ}$ & 0.524 & 0.150 \\
$30^{\circ}$ & 1.531 & 0.245 \\
\hline \hline
\end{tabular}

large energies, one has to be careful with the absolute value.

Inclusion of dynamic correlation at MCCEPA level increased the energy gap between the triplet ground state and the excited quintet state from 55.6 to $86 \mathrm{meV}$. In these calculations the basis set was extended by two $f$ functions at Fe $(3.465,0.843)$, the lone pairs of $\mathrm{N}$, which create the ligand field, the $3 d$ orbitals of Fe, and the singly occupied ligand orbitals were included in the correlation space.

Also, the total energies and exchange coupling constants for HS with additional point charge are listed in Table VIII, fifth column. As expected, the point charge has very little impact on these intrinsic molecular properties.
TABLE X. Zero field splitting of the triplet ground state of $\left[\mathrm{Fe}(\mathrm{HS})(\mathrm{L})_{2}\right]^{0}$ as function of the tilting angle of one ligand. The triplet is split into three levels by spin-orbit coupling, $E_{1}$ and $E_{2}$ are the excitation energies with respect to the ground state. With the parameters $D$ and $E$ the eigenvalues of the traceless $\mathbf{D}$ tensor are given as $-\frac{1}{3} D-E,-\frac{1}{3} D+E, \frac{2}{3} D$.

\begin{tabular}{lcclll}
\hline \hline Angle & $E_{\text {tilt }}(\mathrm{eV})$ & $E_{1}(\mathrm{meV})$ & $E_{2}(\mathrm{meV})$ & $D$ & $E$ \\
\hline $0^{\circ}$ & 0.0 & 0.0 & 0.16 & 0.16 & 0.0 \\
$10^{\circ}$ & 0.03 & 0.28 & 0.84 & 0.70 & 0.14 \\
$20^{\circ}$ & 0.27 & 0.51 & 1.57 & 1.32 & 0.25 \\
$30^{\circ}$ & 0.55 & 0.63 & 2.10 & 1.79 & 0.31 \\
\hline \hline
\end{tabular}

In Table X size and geometry dependency of the zero field splitting in $\left[\mathrm{Fe}(\mathrm{L})_{2}^{-}\right]^{0}$ are given. Because of the large $\mathrm{Fe}-\mathrm{N}$ bond distance the tilting energy is smaller than in the LS compound. The splitting increases with larger angles due to the distortion of the geometry. The ground state degeneracy is removed and the triplet ground state splits into three components. The excitation energy increases and reaches values of the order of the splitting energy of the Kondo peak. *mario.ruben@kit.edu

†ferdinand.evers@kit.edu

${ }^{\ddagger}$ h.s.j.vanderzant@tudelft.nl

${ }^{1}$ J. Park, A. N. Pasupathy, J. I. Goldsmith, C. Chang, Y. Yaish, J. R. Petta, M. Rinkoski, J. P. Sethna, H. D. Abruña, P. L. McEuen, and D. C. Ralph, Nature (London) 417, 722 (2002).

${ }^{2}$ S. Kubatkin, A. Danilov, M. Hjort, H. Cornil, J.-L. Brédas, N. Stuhr-Hansen, P. Hedegård, and T. Bjørnholm, Nature (London) 425, 698 (2003).

${ }^{3}$ A. R. Champagne, A. N. Pasupathy, and D. C. Ralph, Nano Lett. 5, 305 (2005).

${ }^{4}$ Z. K. Keane, J. W. Ciszek, J. M. Tour, and D. Natelson, Nano Lett. 6, 1518 (2006).

${ }^{5}$ E. A. Osorio, K. O’Neill, N. Stuhr-Hansen, O. F. Bjørnholm, and

H. S. J. van der Zant, Adv. Mater. 19, 281 (2007).

${ }^{6}$ N. J. Tao, Phys. Rev. Lett. 76, 4066 (1996).

${ }^{7}$ W. Haiss, T. Albrecht, H. van Zalinge, S. J. Higgins, D. Bethell, H. Höbenreich, D. J. Schiffrin, R. J. Nichols, A. M. Kuznetsov, J. Zhang, Q. Chi, and J. Ulstrup, J. Phys. Chem. B 111, 6703 (2007).

${ }^{8}$ Zh. Li, I. Pobelov, B. Han, Th. Wandlowski, A. Błaszczyk, and M. Mayor, Nanotechnology 18, 044018 (2007).

${ }^{9}$ Y. S. Park, A. C. Whalley, M. Kamenetska, M. L. Steigerwald, M. S. Hybertsen, C. Nuckolls, and L. Venkataraman, J. Am. Chem. Soc. 129, 15768 (2007).

${ }^{10}$ N. Néel, J. Kröger, L. Limot, T. Frederiksen, M. Brandbyge, and R. Berndt, Phys. Rev. Lett. 98, 065502 (2007).

${ }^{11}$ G. Schulze, K. J. Franke, A. Gagliardi, G. Romano, C. S. Lin, A. L. Rosa, T. A. Niehaus, T. Frauenheim, A. Di Carlo, A. Pecchia, and J. I. Pascual, Phys. Rev. Lett. 100, 136801 (2008).

${ }^{12} \mathrm{~S}$. Wu, R. Huber, M. T. Gonzalez, S. Grunder, M. Mayor, C. Schönenberger, and M. Calame, Nat. Nanotechnol. 3, 569 (2008).
${ }^{13}$ M. Ruben, A. Landa, E. Lörtscher, H. Riel, M. Mayor, H. Görls, H. Weber, A. Arnold, and F. Evers, Small 4, 2229 (2008).

${ }^{14}$ C. Li, I. Pobelov, Th. Wandlowski, A. Bagrets, A. Arnold, and F. Evers, J. Am. Chem. Soc. 130, 318 (2008)

${ }^{15}$ For a recent (partial) experimental overview see: D. R. Ward, G. D. Scott, Z. K. Keane, N. J. Halas, and D. Natelson, J. Phys. Condens. Matter 20, 374118 (2008).

${ }^{16}$ R. H. M. Smit, Y. Noat, C. Untiedt, N. D. Lang, M. C. van Hemert, and J. M. van Ruitenbeek, Nature (London) 419, 906 (2002).

${ }^{17}$ H. B. Heersche, Z. de Groot, J. A. Folk, H. S. J. van der Zant, C. Romeike, M. R. Wegewijs, L. Zobbi, D. Barreca, E. Tondello, and A. Cornia, Phys. Rev. Lett. 96, 206801 (2006).

${ }^{18}$ S. Barraza-Lopez, K. Park, V. Garcia-Suarez, and J. Ferrer, Phys. Rev. Lett. 102, 246801 (2009).

${ }^{19}$ J. E. Grose, E. S. Tam, C. Timm, M. Scheloske, B. Ulgut, J. J. Parks, H. D. Abruna, W. Harneit, and D. C. Ralph, Nat. Mater. 7, 884 (2008).

${ }^{20}$ M.-H. Jo, J. E. Grose, K. Baheti, M. M. Deshmukh, and J. J. Sokol, E. M. Rumberger, D. N. Hendrickson, J. R. Long, and H. Park, D. C. Ralph, Nano Lett. 6, 2014 (2006).

${ }^{21}$ P. Gütlich, A. Hauser, and H. Spiering, Angew. Chem. Int. Ed. 33, 2024 (1994).

${ }^{22}$ P. Gütlich, Y. Garcia, and H. A. Goodwin, Chem. Soc. Rev. 29, 419 (2000).

${ }^{23}$ R. F. Klie, J. C. Zheng, Y. Zhu, M. Varela, J. Wu, and C. Leighton, Phys. Rev. Lett. 99, 047203 (2007).

${ }^{24}$ S. Decurtins, P. Gütlich, C. P. Köhler, H. Spiering, and A. Hauser, Chem. Phys. Lett. 105, 1 (1984).

${ }^{25}$ D. Collison, C. Garner, C. M. McGrath, J. F. W. Mosselmans, M. D. Roper, J. M. W. Seddon, E. Sinn, and N. A. Young, J. Chem. Soc. Dalton Trans. 1997, 4371 (1997). 
${ }^{26}$ M. Cavallini, I. Bergenti, S. Milita, G. Ruani, I. Salitros, Z.-R. Qu, R. Chandrasekhar, and M. Ruben, Angew. Chem. Int. Ed. 47, 8596 (2008).

${ }^{27}$ C. Rajadurai, O. Fuhr, R. Kruk, M. Ghafari, and M. Ruben, Chem. Commun. 2007, 2636 (2007).

${ }^{28}$ C. Rajadurai et al., Inorg. Chem. 45, 10019 (2006).

${ }^{29}$ J. J. Parks et al., Science 328, 1370 (2010).

${ }^{30}$ A. Kogan, G. Granger, M. A. Kastner, D. Goldhaber-Gordon, and H. Shtrikman, Phys. Rev. B 67, 113309 (2003); Hauptmann et al., Nat. Phys. 4, 373 (2008).

${ }^{31}$ N. Roch et al., Nature (London) 453, 633 (2008); N. Roch, S. Florens, T. A. Costi, W. Wernsdorfer, and F. Balestro, Phys. Rev. Lett. 103, 197202 (2009).

${ }^{32}$ C. Li, I. Pobelov, T. Wandlowski, A. Bagrets, and F. Evers, J. Am. Chem. Soc. 130, 318 (2008).

${ }^{33}$ A. Bagrets, A. Arnold, and F. Evers, J. Am. Chem. Soc. 130, 9013 (2008).

${ }^{34}$ C. Li, A. Mishchenko, Z. Li, I. Pobelov, T. Wandlowski, F. Würthner, A. Bagrets, and F. Evers, J. Phys. Condens. Matter 20, 374122 (2008).

${ }^{35}$ R. Ahlrichs, M. Bär, M. Häser, H. Horn, and C. Kölmel, Chem. Phys. Lett. 162, 165 (1989).

${ }^{36}$ J. P. Perdew, Phys. Rev. B 33, 8822 (1986).

${ }^{37}$ J. Tao, J. P. Perdew, V. N. Staroverov, and G. E. Scuseria, Phys. Rev. Lett. 91, 146401 (2003); V. N. Staroverov, G. E. Scuseria, J. Tao, and J. P. Perdew, J. Chem. Phys. 119, 12129 (2003).

${ }^{38}$ A. D. Becke, J. Chem. Phys. 98, 5648 (1993).

${ }^{39}$ A. Schäfer, H. Horn, and R. Ahlrichs, J. Chem. Phys. 97, 2571 (1992).

${ }^{40}$ F. Eichkorn, F. Weigend, O. Treutler, and R. Ahlrichs, Theor. Chem. Acc. 97, 119 (1997).

${ }^{41}$ The origin for the functional dependence of the HS-LS energy difference is the large influence of dynamic correlation on the energy gap. On the one hand, dynamic correlation of electron pairs is larger than the dynamic correlation of unpaired electrons. In LS there are three doubly occupied $3 d$ orbitals while there is only one doubly occupied but four singly occupied $3 d$ orbitals in HS. Furthermore, dynamic correlation decreases with increasing distance and the Fe-ligand distance is increased by $10 \%$ in the HS state.

${ }^{42}$ A. Bernard-Mantel, J. S. Seldenthuis, A. Beukman, H. S. J. van der Zant, V. Meded, R. Chandrasekhar, K. Fink, M. Ruben, and F. Evers, e-print arXiv:1004.4556v1.

${ }^{43}$ U. Meier and V. Staemmler, Theor. Chim. Acta 76, 95 (1989).

${ }^{44}$ J. Wasilewski, Int. J. Quantum Chem. 36, 503 (1989).

${ }^{45}$ R. Fink and V. Staemmler, Theor. Chim. Acta 87, 129 (1993).

${ }^{46}$ N. Suaud, M.-L. Bonnet, C. Boilleau, P. Labguerie, and N. Guihry, J. Am. Chem. Soc. 131, 715 (2009).

${ }^{47}$ M. Kepenekian, V. Robert, B. Le Guennic, and C. De Graaf, J. Comput. Chem. 30, 2327 (2009).

${ }^{48}$ K. Fink, C. Wang, and V. Staemmler, Inorg. Chem. 38, 3847 (1999).

${ }^{49}$ F. Neese, J. Chem. Phys. 122, 034107 (2005).

${ }^{50}$ Yu. Ralchenko, A. E. Kramida, J. Reader, and NIST ASD Team (2011), NIST Atomic Spectra Database (ver. 4.1.0) [http://physics.nist.gov/asd3] National Institute of Standards and Technology, Gaithersburg, MD, See also: J. Sugar and C. Corliss, J. Phys. Chem. Ref. Data 14, Suppl. 2, 1664 (1985).

${ }^{51}$ S. Koseki, M. W. Schmidt, and M. S. Gordon, J. Phys. Chem. A 102, 10430 (1998).
${ }^{52}$ J. Paaske, A. Rosch, P. Wölfle, N. Mason, C. M. Marcus, and J. Nygard, Nat. Phys. 2, 460 (2006).

${ }^{53}$ K. O'Neill, E. A. Osorio, and H. S. J. van der Zant, Appl. Phys. Lett. 90, 133109 (2007).

${ }^{54}$ E. A. Osorio, T. Bjørnholm, J.-M. Lehn, M. Ruben, and H. S. J. van der Zant, J. Phys. Condens. Matter 20, 374121 (2008).

${ }^{55}$ D. Goldhaber-Gordon, J. Göres, M. A. Kastner, H. Shtrikman, D. Mahalu, and U. Meirav, Phys. Rev. Lett. 81, 5225 (1998).

${ }^{56}$ N. Roch, S. Florens, T. A. Costi, W. Wernsdorfer, and F. Balestro, Phys. Rev. Lett. 103, 197202 (2009).

${ }^{57}$ A. Eliasen, J. Paaske, K. Flensberg, S. Smerat, M. Leijnse, M. R. Wegewijs, H. I. Jorgensen, M. Monthioux, and J. Nygard, Phys. Rev. B 81, 155431 (2010).

${ }^{58}$ H. Jeong, A. M. Chang, and M. R. Melloch, Science 293, 2221 (2001).

${ }^{59}$ N. J. Craig, J. M. Taylor, E. A. Lester, C. M. Marcus, M. P. Hanson, and A. C. Gossard, Science 304, 565 (2004).

${ }^{60}$ R. Aguado and D. C. Langreth, Phys. Rev. Lett. 85, 1946 (2000).

${ }^{61}$ M. G. Vavilov and L. I. Glazman, Phys. Rev. Lett. 94, 086805 (2005).

${ }^{62}$ Ch. Stroh, Ph. Turek, P. Rabu, and R. Ziessel, Inorg. Chem. 40, 5334 (2001).

${ }^{63}$ Our $a b$ initio calculations indicates that the splitting between ground state triplet and the consecutive quintet is at least $53.2 \mathrm{meV}$ corresponding to a metal ion-ligand exchange interaction $J_{i}^{\mathrm{Fe}-\mathrm{L}} \approx$ $-13.3 \mathrm{meV}$, in the same range as the one experimentally observed in similar transition metal complex. ${ }^{62,64}$

${ }^{64}$ E. A. Osorio et al., Nano Lett. 10, 105 (2010).

${ }^{65}$ L. Bogani and W. Wernsdorfer, Nat. Mater. 7, 179 (2008).

${ }^{66}$ Incidentally, we can even detect a weak violation of the perfect octahedral symmetry in $\mathbf{1}$. It manifests itself in a small splitting $\Delta_{t, e} \approx 60 \mathrm{meV}$ of the $t_{2 g}$ triplet and the $e_{g}$ doublet.

${ }^{67}$ J. Elhaiik, D. J. Evans, C. A. Kilner, and M. A. Halcrow, Dalton Trans. 2005, 1693 (2005).

${ }^{68}$ J. M. Holland, C. A. Kilner, M. Thornton-Pett, M. A. Halcrow, J. A. McAllister, and Zh. Lu, Chem. Commun. 2001, 577 (2001).

${ }^{69}$ H. Oshio, H. Spiering, V. Ksenofontov, F. Renz, and P. Gütlich, Inorg. Chem. 40, 1143 (2001).

${ }^{70}$ J. M. Holland, J. A. McAllister, C. A. Kilner, M. Thornton-Pett, A. J. Bridgeman, and M. A. Halcrow, J. Chem. Soc. Dalton Trans. 2002, 548 (2002).

${ }^{71}$ P. S. Braterman, J. I. Song, and R. D. Peacock, Inorg. Chem. 31, 555 (1992).

${ }^{72}$ D. L. Jameson, J. K. Blaho, K. T. Kruger, and K. A. Goldsby, Inorg. Chem. 28, 4312 (1989).

${ }^{73}$ We ignore at this point the spin-orbit interaction. This is appropriate for the interpretation of our numerical data, since our DFT calculation ignores relativistic effects as well. For a better comparison of theoretical spectra with experimental data, we plan to investigate the effect of spin-orbit coupling in future work.

${ }^{74}$ A. H. J. Wachters, J. Chem. Phys. 52, 1033 (1970).

${ }^{75}$ S. Huzinaga, Approximate Atomic Functions. I and II. Preprints (University of Alberta, Alberta, Canada, 1971). 